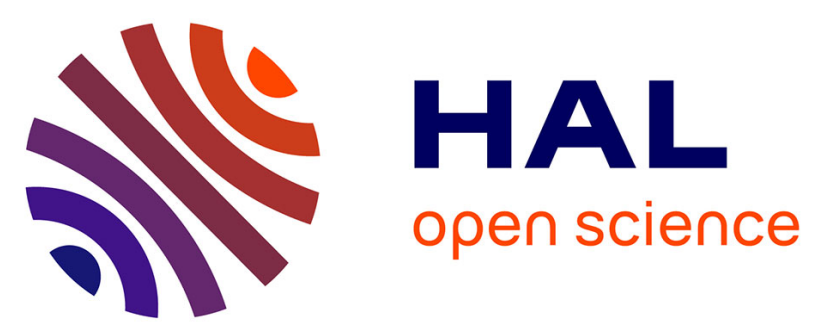

\title{
Dynamics of the semi-diurnal and quarter-diurnal internal tides in the Bay of Biscay. Part 1: Barotropic tides
}

\author{
Ivane Lilian Pairaud, Florent Lyard, Francis Auclair, Thierry Letellier, \\ Patrick Marsaleix
}

\section{To cite this version:}

Ivane Lilian Pairaud, Florent Lyard, Francis Auclair, Thierry Letellier, Patrick Marsaleix. Dynamics of the semi-diurnal and quarter-diurnal internal tides in the Bay of Biscay. Part 1: Barotropic tides. Continental Shelf Research, 2008, 28 (10-11), pp.1294-1315. 10.1016/j.csr.2008.03.004 . hal02110215

\section{HAL Id: hal-02110215 \\ https://hal.science/hal-02110215}

Submitted on 10 Aug 2021

HAL is a multi-disciplinary open access archive for the deposit and dissemination of scientific research documents, whether they are published or not. The documents may come from teaching and research institutions in France or abroad, or from public or private research centers.
L'archive ouverte pluridisciplinaire HAL, est destinée au dépôt et à la diffusion de documents scientifiques de niveau recherche, publiés ou non, émanant des établissements d'enseignement et de recherche français ou étrangers, des laboratoires publics ou privés. 


\title{
Dynamics of the semi-diurnal and quarter-diurnal internal tides in the Bay of Biscay. Part 1: Barotropic tides
}

\author{
I.L. Pairaud ${ }^{\text {a,* }}$, F. Lyard ${ }^{\text {b }}$, F. Auclair ${ }^{\text {a }}$, T. Letellier ${ }^{\text {b }}$, P. Marsaleix ${ }^{\text {a }}$ \\ a Laboratoire d'Aérologie, UMR 5560, Observatoire Midi-Pyrénées, 14 av. Edouard Belin, 31400 Toulouse, France \\ ${ }^{\mathrm{b}}$ LEGOS, UMR 5566, Observatoire Midi-Pyrénées, 18 av. Edouard Belin, 31400 Toulouse, France
}

\begin{abstract}
The generation of internal tides in the ocean is due to the interaction of strong barotropic tidal currents with variable topography in stratified waters, transferring energy from the external to the deep ocean. The internal tides feed later the ocean mixing, playing a major role for the maintenance of the stratification of the global ocean. A remarkable region in terms of tidal energy is the European continental shelf. As a first step toward the study of internal tides in the Bay of Biscay, this paper aims at understanding the barotropic tides and associated energy budgets. On continental shelves and in coastal seas the use of regional models with fine grid resolution is preferred to the use of global tidal atlases derived from altimetry. The unstructured grid T-UGOm model is used to compute the NEA-2004 tidal solutions in the North-East Atlantic ocean, with errors greatly reduced in coastal areas compared with global models. Energy budgets are discussed based on the inclusion of nonlinearities in the tidal solutions. The sea surface height and depth-averaged currents are used to compute the tidal energy conversion from barotropic to baroclinic tides, tidal dissipation and energy flux. A total amount of energy of $250 \mathrm{GW}$ is found for the $M_{2}$ tide. The path of $M_{4}$ energy from the Southern Atlantic ocean toward the Bay of Biscay is highlighted, advocating for nonzero boundary conditions in regional models. The 3D coastal ocean SYMPHONIE model has been implemented to simulate the surface tides in the Bay of Biscay. Solutions are validated by comparison with the NEA-2004 solutions and observations.
\end{abstract}

\section{Introduction}

The history of tidal dynamical science has recently been driven by two major revolutions: numerical modeling and satellite altimetry. Shallow-water barotropic models have so far been extremely successful at simulating the barotropic tides, and until the late 1990s the internal tides were generally seen as a marginal process with little influence on the first order, barotropic component. Still, the problem of energy conversion from the barotropic mode to the baroclinic modes has drawn the attention of several authors. Bell (1975) first estimated the conversion rate to reach $10 \%$ of the total tidal energy in the case of the $M_{2}$ tide, a value revised upward by Cartwright (1977) who found 25\%, close to more recent estimates of $\frac{1}{3}$ (Garrett and Kunze, 2007). From the approximately 3.5 terawatts (TW) of tidal energy dissipated in the ocean, Munk and Wunsch (1998) pointed out that internal tides could provide about $1 \mathrm{TW}$ missing in the ocean mixing necessary

\footnotetext{
* Corresponding author. Tel.: +33476825045; fax: +33476825271.

E-mail addresses: ivane.pairaud@gmail.com (I.L. Pairaud),

Florent.Lyard@notos.cst.cnes.fr (F. Lyard), francis.auclair@aero.obs-mip.fr (F. Auclair), patrick.marsaleix@aero.obs-mip.fr (P. Marsaleix).
}

to sustain the ocean abyssal circulation and global overturning. The examination of the tidal energy balance in global barotropic models has repeatedly shown a lack of tidal energy dissipation that could not be compensated by classical bottom friction. Satellite altimetry finally demonstrated the widespread existence of internal tides all over the global ocean. It is now admitted that the barotropic/baroclinic energy conversion is one of the major dissipation mechanisms of the barotropic tide. Simple parameterization of this conversion allows to improve greatly the accuracy of the global barotropic tidal models (Egbert et al., 2004; Lyard et al., 2006). The energy converted into internal waves is thought to be partly available for ocean mixing where the waves dissipate. The mechanism of dissipation, either at the region of generation of the waves or in remote regions after their propagation, is complex and not entirely understood. Once again, a rough parameterization based on an increase in the vertical diffusion coefficient in regions of generation of internal tides can account partly for the ocean mixing, and improve the overturning of the global ocean circulation (Simmons et al., 2004). In continental shelf regions, the increase in bottom turbulence caused by internal tides will impact the sediment transport and more generally the exchange between the shelf and the deep ocean. It will also impact vertical nutrient transport with a consequence for the phytoplancton growth 
depending on the lighting. In short, ocean sciences have entered a time when the interaction of the tides with the ocean stratification and circulation cannot be ignored any longer, from climate change scales to coastal ocean dynamics scales. So far, the hydrodynamic models have mostly been specialized to the resolution of a limited range (both in frequency and horizontal scales) of the ocean dynamics. The comprehensive modeling of the internal tide dynamics requires simultaneous resolution of the full range of the ocean dynamics, and therefore remains a very challenging task.

It is the objective of this study to precisely investigate and improve the understanding of tidal dynamics at a regional scale. Because of the large amplification of the tides on the Northeast European shelf, and the tidal energy flux toward the English Channel, the Bay of Biscay is a place of intense internal tide production over the shelf break, as has been observed for example by Pingree et al. (1986). In addition, altimetric and in situ data are available for observations of barotropic and internal tides and model validations (Lyard et al., 2006; Pichon and Correard, 2006). For those reasons, the Bay of Biscay is an ideal location to study the tidal dynamics, and to assess numerical model skills.

As a first step, the first part of the paper deals with the barotropic tide modeling. From previous studies, the dominant component of the tides is the semi-diurnal lunar $M_{2}$ wave which is responsible for the low tide-high tide cycle. Diurnal and semidiurnal tides are known to propagate principally as Kelvin waves (Le Cann, 1990) in the Bay of Biscay with maximum amplitudes at the oceanic boundaries. The surface elevation due to the tide is typically $\mathrm{O}(0.2 \mathrm{~m})$ in the ocean and $\mathrm{O}(1 \mathrm{~m})$ near the coasts. The principal quarter-diurnal component is the $M_{4}$ wave, which does not have an astronomical origin but is a nonlinear wave resulting from the interaction of the $M_{2}$ wave with itself. Its contribution is negligible in the deep ocean, but the same is not true in the coastal ocean. On the shelf of the Bay of Biscay, the amplitude of the $M_{4}$ surface elevation reaches several tens of centimeters as seen from altimetric data (Andersen, 1999); accordingly, it has to be modeled accurately before the quarter-diurnal internal tides are investigated.

In the first section, the 2D North-East Atlantic tidal dynamics is modeled using the barotropic finite-element T-UGOm model. Validation is carried out against observations. Important tidal features are observed, in particular the amplification of the tides at the coast with a focus on the $M_{2}$ wave and the $M_{4}$ harmonic. The present NEA-2004 tidal atlas includes the nonlinear tidal dynamics. It is more accurate than the FES2004 atlas from Lyard et al. (2006) over continental shelves, especially in coastal regions, which is of particular interest for internal tide studies. The related energy budgets give the opportunity to better understand the tidal dynamics and the possible sources for ocean mixing. Moreover the budget balance assesses the consistency of the model. Attention is paid to the presence of nonlinearities, in particular within the energy budgets, and the departure from the spectral linear approach is discussed.

The second section is devoted to the validation of the tidal solutions obtained with the 3D finite-difference SYMPHONIE model (Marsaleix et al., 2008) embedded into the T-UGOm regional model. This is the first step toward the modeling of internal tides in the Bay of Biscay, described in Part 2 of the paper.

\section{The NEA-2004 barotropic tidal atlas}

Although the global tidal atlases, such as the FES2004 atlas (Lyard et al., 2006), perform extremely well in the deep ocean, their accuracy tends to degrade in the shelf and coastal waters, and their spatial resolution is no longer adequate. The derivation of the tidal currents from the satellite altimetry empirical or assimilated global models is an uneasy task. The assimilated tidal current solution relies directly on the model error description, since only elevation data are assimilated. Given that such a description remains extremely empirical and rough in the global tidal models, the question of our confidence in such derivation remains controversial, especially in the shelf and coastal waters. For these reasons, and like most of the available global assimilating models, the FES2004 atlas provides only the tidal elevations, not the tidal currents. Furthermore, the amplitude of the compound tides is greatly amplified over the continental shelf and cannot be disregarded from the tidal predictions. It is for instance the case for the $M_{4}$ tide, which exhibits an amplitude larger than $10 \mathrm{~cm}$. In the English Channel, the $M_{4}$ amplitude is larger than the amplitude of the main diurnal tides. Lifting the accuracy of global models at the same level in shelf and deep ocean regions will still require significant further developments. At present, the best strategy consists in nesting regional and shelf tidal models into the global models. Accordingly, the North-East Atlantic (NEA-2004) tidal atlas has been obtained in order to provide a regional tidal atlas with high accuracy, high resolution and extended tidal spectrum.

\subsection{Model setup}

The NEA-2004 tidal atlas has been analyzed from a one yearlong tidal simulation, carried out using the Toulouse Unstructured Grid Ocean model (T-UGOm) in a 2D barotropic shallow-water mode. T-UGOm (which is the follow-up of MOG2D) is based on an unstructured mesh which allows the resolution to be increased in regions of particular interest, and allows a more realistic description of the shorelines. In the NEA application, the T-UGOm 2D model has been run in a $P_{1} \times P_{1}$ mode solving the generalized wave equation with a finite-element discretization. This configuration is identical to the MOG2D generalized wave equation solver that has been derived from Lynch and Gray (1979). The NEA-2004 mesh resolution (Fig. 1) has been constrained by the typical horizontal scale of the tidal distributions (like the tidal wavelength for elevation, and the $2 \pi H / H^{\prime}$ scale for the barotropic currents). Typically the local resolution is set to $\frac{1}{15}$ of the shortest horizontal scale. It ranges from $20 \mathrm{~km}$ over the deep ocean to less than $1 \mathrm{~km}$ along the coastlines. Over the continental shelf, the resolution ranges from 1 to $10 \mathrm{~km}$ and is about $3 \mathrm{~km}$ over the shelf break. The bathymetry is of course a critical parameter for the tidal modeling, and must be set with great care. Unfortunately no homogeneous quality database exists for the North-East Atlantic region, and consequently the model bathymetry has been built by merging several databases, such as DBDB, GEBCO, Etopo2, Smith and Sandwell, and declassified French Navy's terrain models. The boundary conditions in elevation have been extracted from the FES2004 global model. The model is forced by the astronomical plus the loading and self-attraction (LSA) potentials. The LSA potential is computed from the FES2004 atlas on a $\frac{1}{8}^{\circ}$ resolution structured grid (Lyard et al., 2006). As this is a depth-averaged barotropic model, a parameterization (Lyard et al., 2006) is employed in order to account for the internal wave drag.

\subsection{Tidal solutions}

Thirteen tidal constituents (i.e. the 12 major astronomic constituents plus the $M_{4}$ tide) have been used to impose the open boundary conditions (OBC) in elevation: $M_{4}, M_{2}, S_{2}, N_{2}, K_{2}$, $2 N_{2}, K_{1}, O_{1}, P_{1}, Q_{1}, M_{f}, M_{m}, M_{t m}$. The constituent's list taken for the boundary conditions has been extended with the minor astronomic constituents such as $M u_{2}$ or $L_{2}$ by using the 


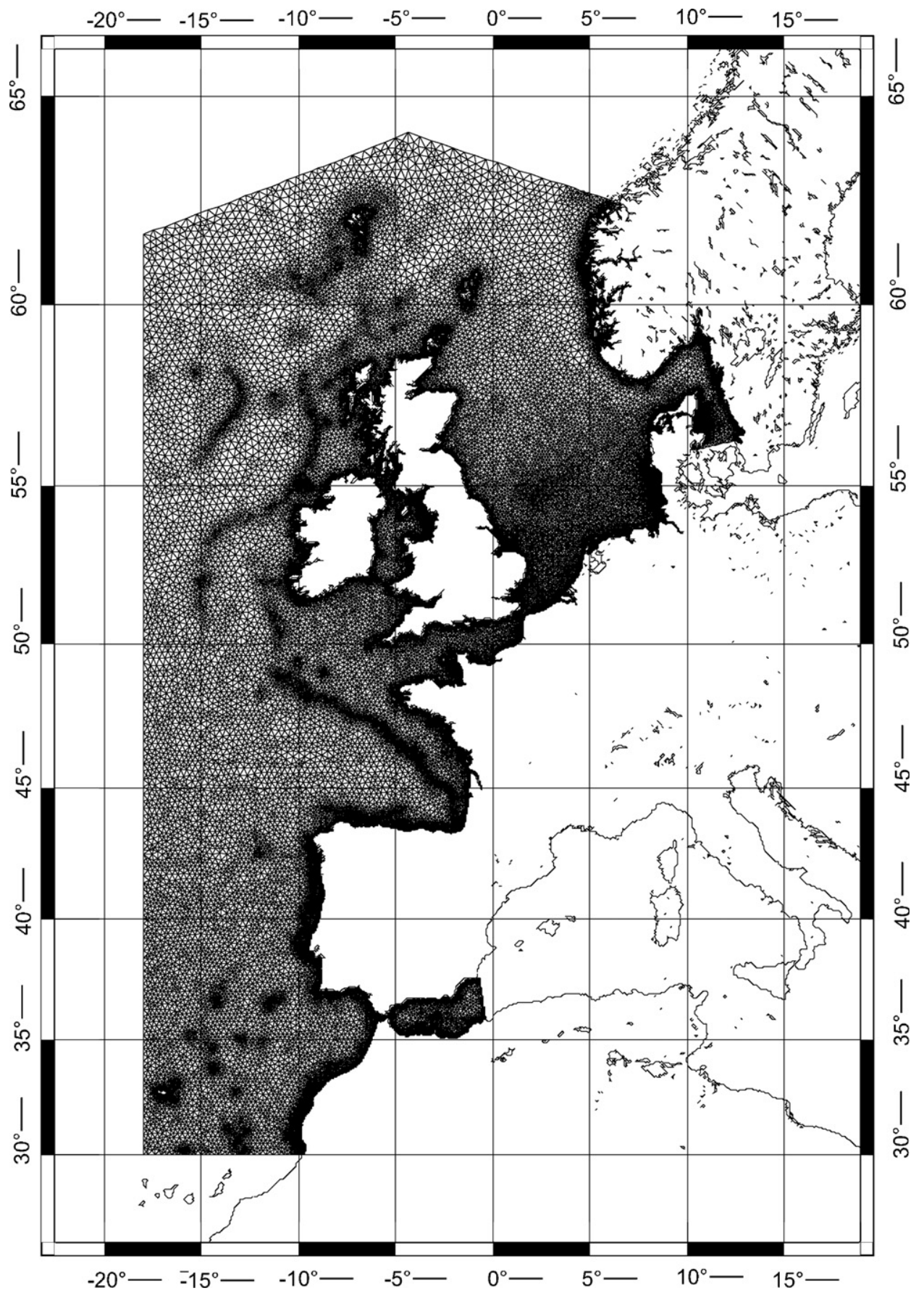

Fig. 1. NEA-2004 finite-element mesh.

admittance method (Munk and Cartwright, 1966). Forty tidal constituents, namely the forcing tides plus the major overtides and compound tides, including the permanent terms, have been harmonically analyzed (Schureman, 1958) from the hourly simulated time series. Finally the tidal elevations have been fully validated against in situ and satellite altimeter data analysis. The NEA-2004 atlas (harmonic elevation and barotropic currents) can be obtained by sending a request at Florent.Lyard@cnes.fr. To avoid a tedious presentation of the model results and accuracy, we will restrict ourselves in the following to the most representative constituents. As an illustration, we have displayed the tidal distribution of the $M_{2}$ and $M_{4}$ tidal waves, i.e. the major tidal constituent in the North-East Atlantic ocean and its first harmonic. The $M_{2}$ cotidal charts (Figs. 2 and 3) show the characteristic amphidromic system (described in Le Provost and Fornerino, 1985; Davies and Kwong, 2000), in which the main items are the Isle of Wight's amphidrom (English Channel) and the triple amphidroms of the North Sea. The $M_{4}$ amplitude (Fig. 4) is significantly above $2 \mathrm{~cm}$ only in the shelf seas, with a maximum of $40 \mathrm{~cm}$ reached in the English Channel. The amphidrom distribution (Fig. 5) illustrates the shorter wavelength of $M_{4}$ (half that of $M_{2}$ ). The comparisons between data and model solutions are given in Table 1 for the major tidal components, and the overall scores in Tables $2-4$. We have also reported the validation figures of the empirical model GOT00 and assimilated model FES2004 as an additional comparison. As should be expected, the NEA-2004 regional model shows a slightly degraded accuracy compared with the GOTO0 and FES2004 models in the open ocean 


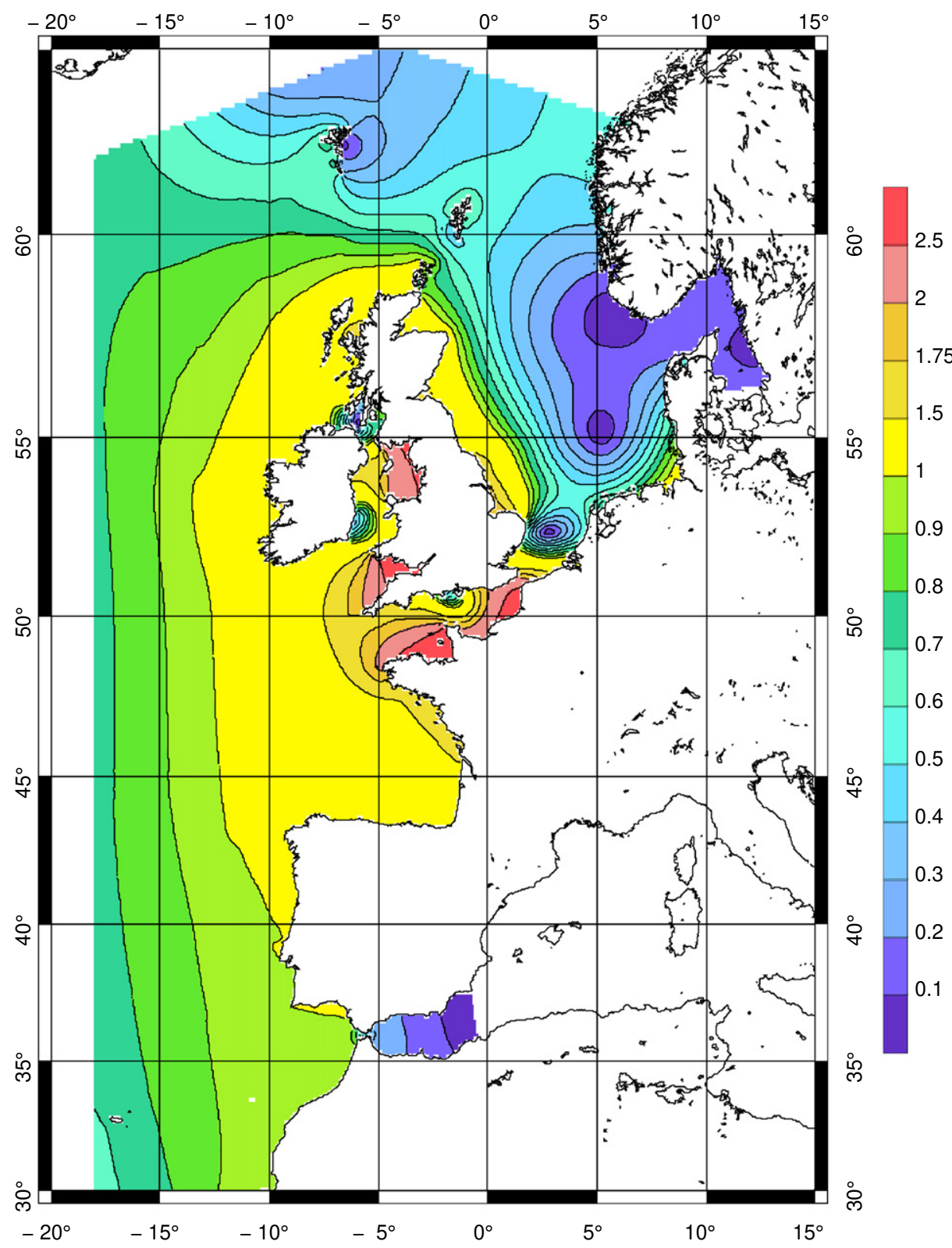

Fig. 2. Amplitude of the $M_{2}$ tide computed from T-UGOm 2D. Units in meters.

and shelves (remember that the regional model is not constrained with data except at the open boundaries), but shows conversely a significantly better accuracy in most of the coastal zones. The largest discrepancy takes place in the English Channel. Of course, this discrepancy is amplified locally in proportion to the very high tidal amplitudes. Nevertheless we suspect that the uncertainties over the bathymetry of the model and their impact on the propagation of the tidal wave in the shallow water of the Channel are responsible for a great deal of additional error in the solutions. Despite some efforts to compile additional data in the digital database, the accuracy of the model bathymetry must be considered as insufficient in this area. It is also well-known that the coefficient of bottom friction depends on the local nature of the seabed, and its variation can have a strong impact on the tidal amplitudes. In the case of the NEA-2004 atlas, the model friction coefficient has been taken as uniform (equal to $2.5 \times 10^{-3}$ ) and this might also explain the relatively poor local performances of the model in the English Channel, and more generally in the North Sea shelf.

\subsection{Barotropic energy budget}

The energy budget of the barotropic tides is a highly valuable tool for investigating and understanding the tidal dynamics. It is also a practical test for model consistency. We will consider in this section the tidal energy issue and again we shall focus on the $M_{2}$ and $M_{4}$ waves. The instantaneous tidal energy is huge and mostly periodic. Despite being typically at least two orders of magnitude smaller than the instantaneous tidal energy, the mean tidal energy (i.e. averaged over a tidal period if considering a single constituent) is far more interesting, since it quantifies the permanent effective characteristics of the tidal dynamics: generation, propagation and dissipation. The gravitational forces feed the astronomical tides mostly in the deep ocean (Le Provost and Lyard, 1998). From there the energy propagates in the ocean toward the energy dissipation regions, where the bottom layer turbulence (in the shelf and coastal seas) or the internal tide excitation (above steep 


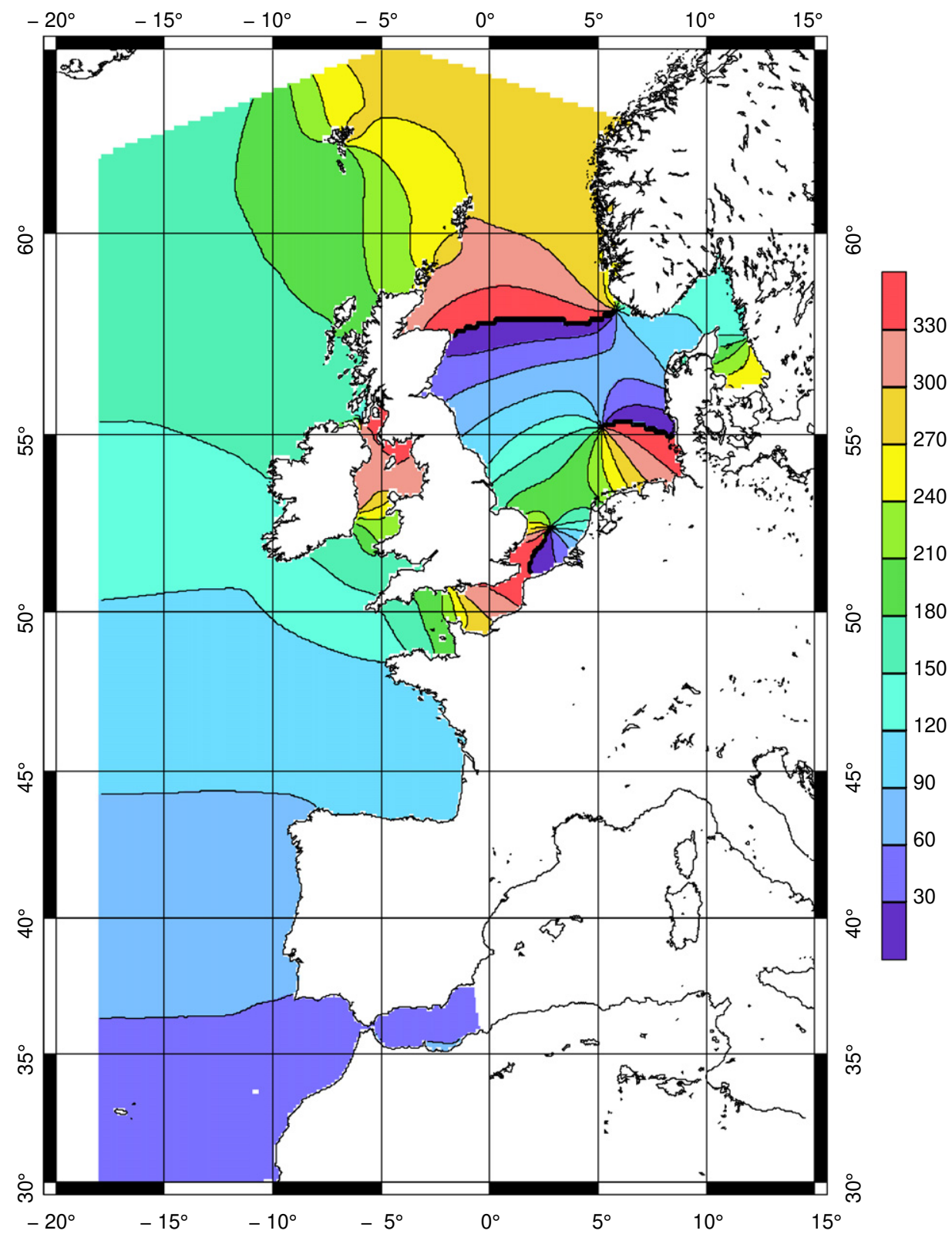

Fig. 3. Phase lag of the $M_{2}$ tide computed from T-UGOm 2D. Units in degrees.

bathymetries) will substract energy from the barotropic tides. The energy can travel along great distances before being damped. For instance, a great deal of the $M_{2}$ energy brought to the ocean in the South Atlantic will dissipate in the North Atlantic and the Hudson Bay. To some extent, the tidal dynamics reveals a world-wide network. Conversely, the compound tides are generated by the nonlinear dynamics of the astronomical tides where the tidal currents are substantial, i.e. in the shallow waters. Consequently, they are generated in the vicinity of the energy dissipation regions and experience very little propagation away from there. The only exception known by the authors is the $M_{4}$ energy propagation from the Patagonian shelf toward the South Atlantic open ocean, and the Amazonian and European shelf seas (Fig. 6). The consequence of this remarkable exception is the necessity to prescribe deep ocean boundary conditions for the $M_{4}$ wave regional modeling in the abovementioned seas.

\subsubsection{Energy equations}

The partition of the mean tidal energy between the contributions of all the constituents (spectral mean energy budget) is a usual approach in tidal science. The mean spectral energy derivation is straightforward when considering the linear or quasi-linear tidal equations, and most of the tidal energy estimates produced in literature have been obtained in this way. As far as the main astronomical tidal constituents are concerned, these estimates can be considered fairly accurate. In order to compare our results with them, the mean energy budget of the NEA-2004 simulation has been partitionned between the various tidal constituents. However, the justification and the computation of the spectral mean energy budget from a fully nonlinear timestepping model is not straightforward and some additional derivations are necessary. In the following, we will derive the various terms involved in the mean energy budget with the objective to remain as consistent as possible with the linear 


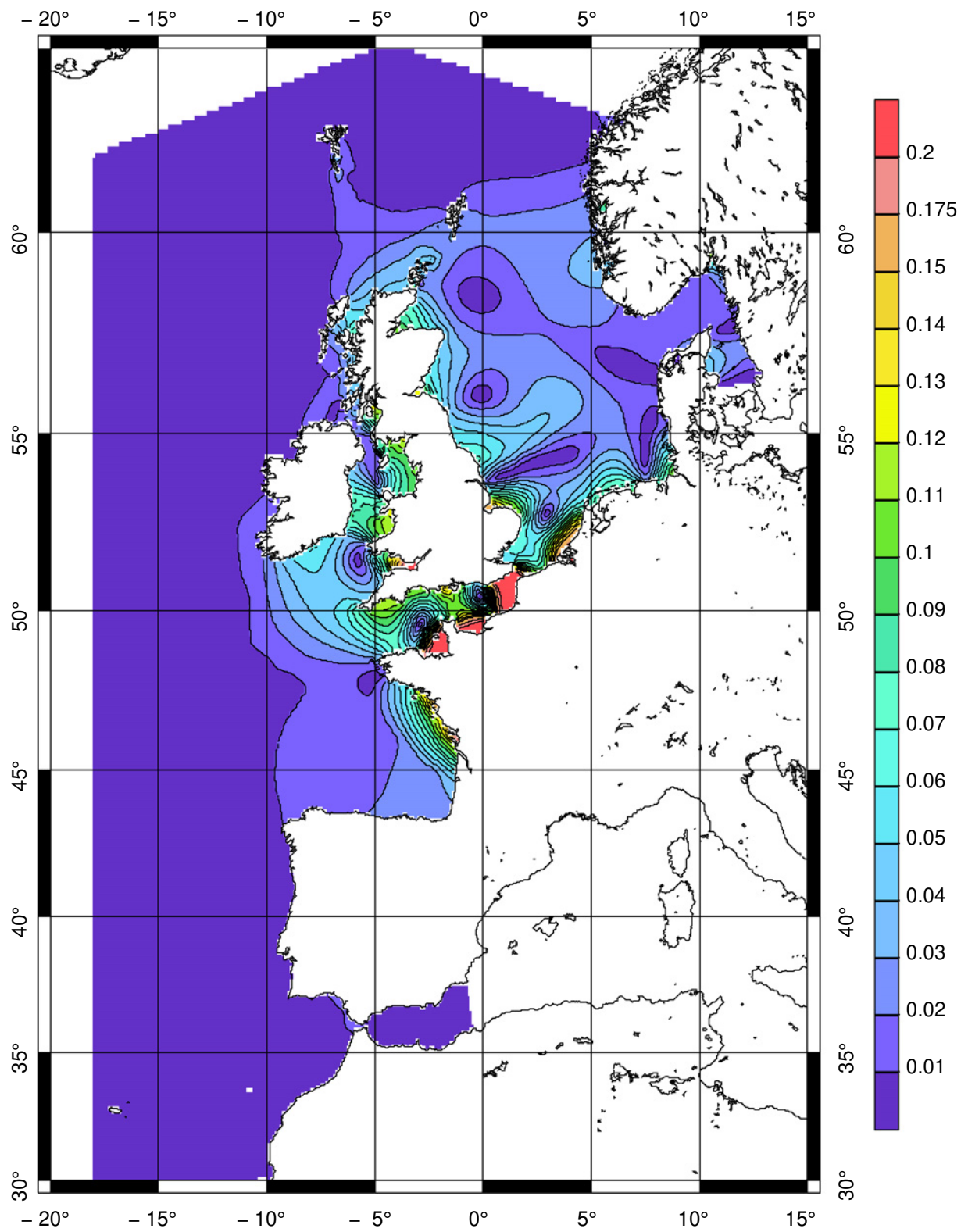

Fig. 4. Amplitude of the $M_{4}$ tide computed from T-UGOm 2D. Units in meters.

approach, and to highlight the main departure from this approach. We start from the momentum equation, namely:

$\frac{\partial \mathbf{u}}{\partial t}+\mathbf{u} \cdot \nabla \mathbf{u}+2 \mathbf{\Omega}^{\prime} \otimes \mathbf{u}=-g \nabla \eta+\mathbf{F}+\mathbf{D}$

where

$\mathbf{F}=\left(1+k_{2}-h_{2}\right) g \nabla \Pi_{a}+g \nabla \Pi_{L S A}$

$\mathbf{D}=\nabla \cdot(A \nabla \mathbf{u})-\frac{C}{H}\|\mathbf{u}\| \mathbf{u}-c(\nabla h \cdot \mathbf{u}) \nabla h$

and the 2D continuity equation:

$\frac{\partial \eta}{\partial t}+\nabla \cdot(H \mathbf{u})=0$

with $\mathbf{u}$ the total barotropic current, $\eta$ the sea surface elevation, $H$ the total water depth, $h$ the mean water depth $(H=\eta+h), g$ the gravitational acceleration, $\boldsymbol{\Omega}^{\prime}$ the Coriolis and metrics contribution, $k_{2}$ and $l_{2}$ the potential and deformation Love numbers, $\Pi_{a}$ the astronomical potential, $\Pi_{L S A}$ the LSA potential, $A$ the horizontal momentum diffusion coefficient, $C$ the quadratic friction coefficient, $c$ the internal wave drag coefficient. The last term in Eq. (3) represents the conversion of barotropic momentum into baroclinic modes. Recent studies have shown that this energy sink represents about one-third of the global $M_{2}$ barotropic tidal dissipation, and is made available partly for ocean mixing increase (Lyard et al., 2006). Following the consequent renewed interest for the internal tides, the parameterization of this mechanism has been widely addressed in the past five years. Our formulation is designed to account for the conversion occurring above the main topographic slopes, such as the shelf edges or the volcanic ridges. The coefficient $c$ is proportional to a typical horizontal tidal excursion and to a typical first moderelated buoyancy frequency. It must also account for the lack of resolution in the model topography gradient, and has been tuned empirically (with respect to error misfits minimization and/or energy budget consistency). We can derive the kinetic energy 


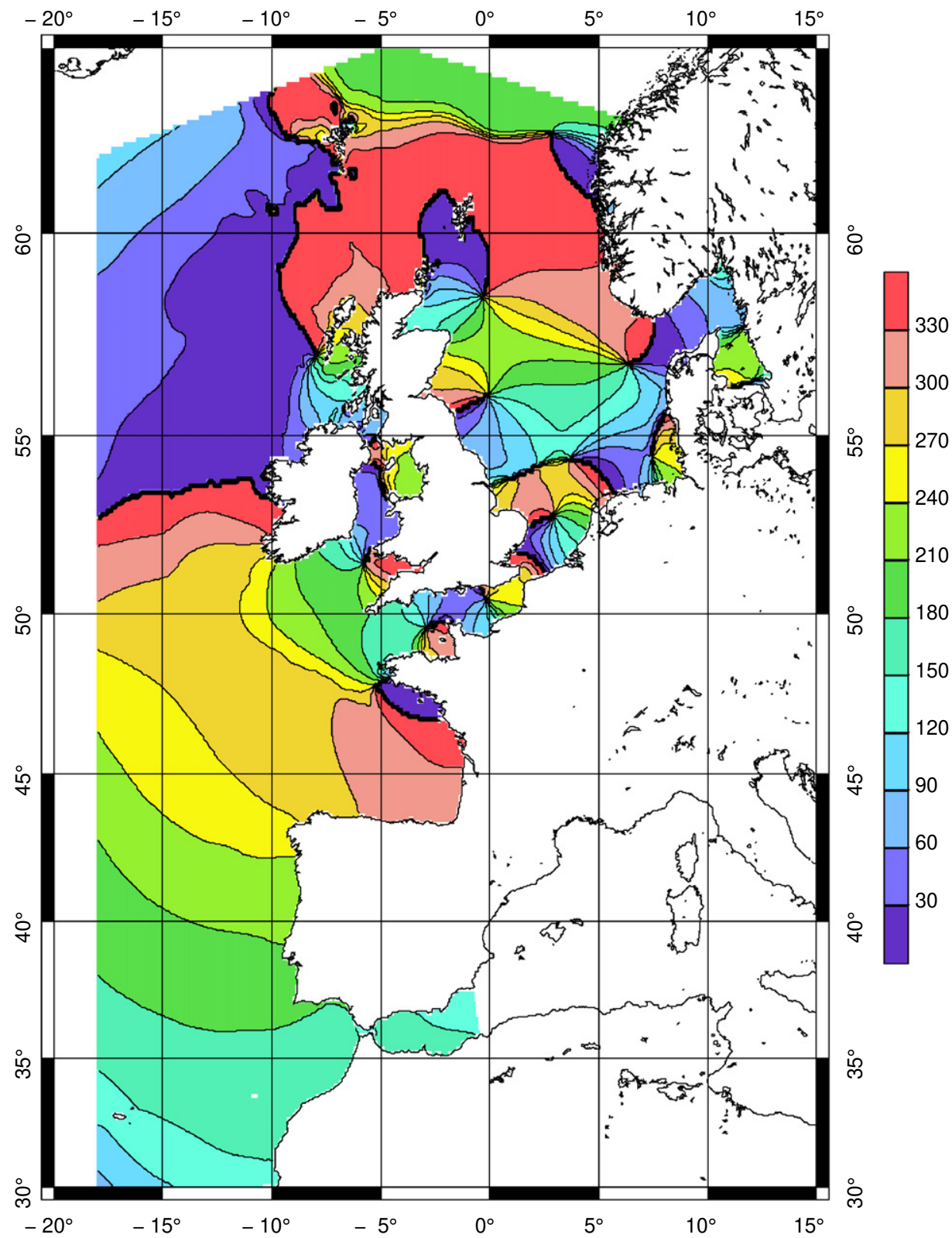

Fig. 5. Phase lag of the $M_{4}$ tide computed from T-UGOm 2D. Units in degrees.

equation by multiplying Eq. (1) by $\rho H \mathbf{u}$ and using Eq. (4):

$$
\begin{aligned}
\frac{\partial e_{k}}{\partial t}+\nabla \cdot e_{k} \mathbf{u}= & -\rho g H \mathbf{u} \cdot \nabla \eta \\
& +\rho H \mathbf{u} \cdot \mathbf{F}+\rho H \mathbf{u} \cdot \mathbf{D}
\end{aligned}
$$

where $e_{k}$ is the kinetic energy per surface unit

$e_{k}=\frac{1}{2} \rho H \mathbf{u} \cdot \mathbf{u}$

Terms in Eq. (5) are periodic and the permanent component of the energy budget is obtained by averaging over a tidal period. In fact, because of the multi-periodic nature of the tides, one would consider the time-averaged energy budget over a large time interval. When the simulation is stabilized (i.e. nondivergent), the kinetic energy content remains bounded and therefore:

$\lim _{T \rightarrow \infty} \frac{1}{T} \int_{0}^{T} \frac{\partial e_{k}}{\partial t} \mathrm{~d} t=\lim _{T \rightarrow \infty} \frac{1}{T}\left[e_{k}\right]_{0}^{T}=0$ and the time-averaged energy budget is given by

$\lim _{T \rightarrow \infty} \frac{1}{T} \int_{0}^{T} \nabla \cdot\left(e_{k} \mathbf{u}\right) \mathrm{d} t=\bar{w}_{p}+\bar{w}_{\mathbf{F}}+\bar{w}_{\mathbf{D}}$

where

$\bar{w}_{p}=-\lim _{T \rightarrow \infty} \frac{1}{T} \rho g \int_{0}^{T} H \mathbf{u} \cdot \nabla \eta \mathrm{d} t$

$\bar{w}_{\mathbf{F}}=\lim _{T \rightarrow \infty} \frac{1}{T} \rho \int_{0}^{T} H \mathbf{u} \cdot \mathbf{F} \mathrm{d} t$

$\bar{w}_{\mathbf{D}}=\lim _{T \rightarrow \infty} \frac{1}{T} \rho \int_{0}^{T} H \mathbf{u} \cdot \mathbf{D} \mathrm{d} t$

The energy advection term can usually be considered negligible, at least for the main astronomical constituents, implying that to the first order, the time-averaged local production and dissipation of energy are balanced by the time-averaged rate of work (RoW) of 


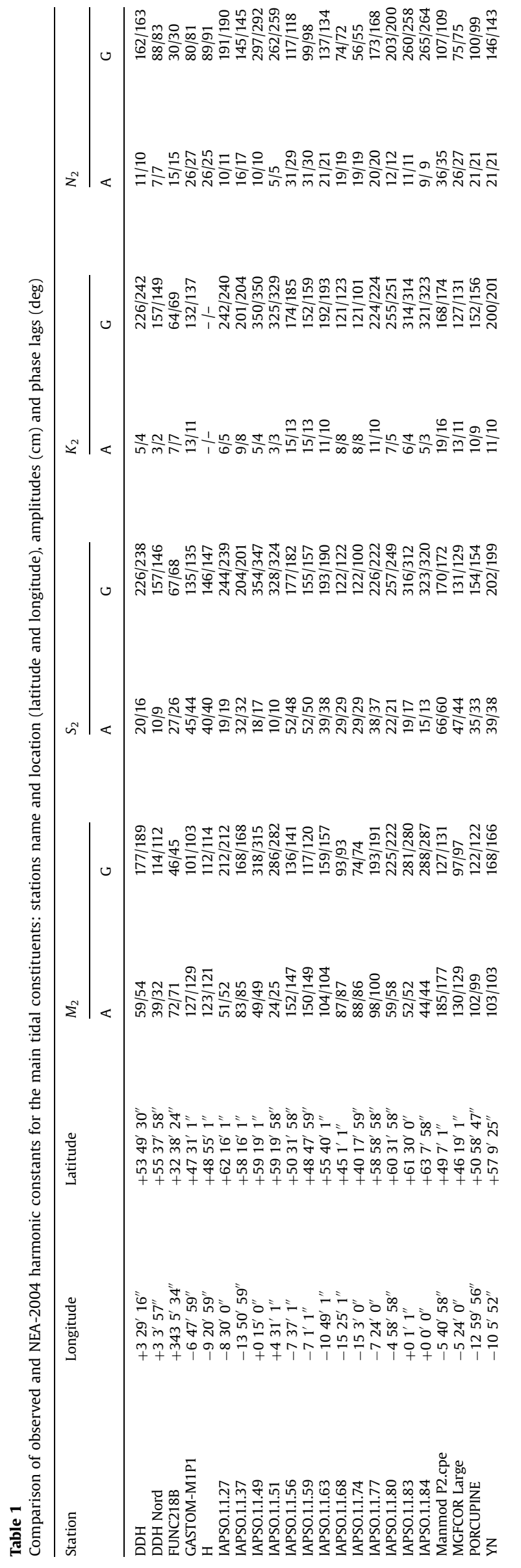

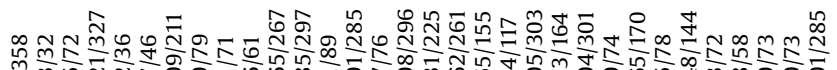

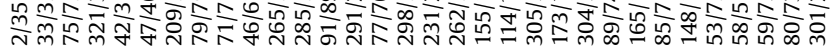

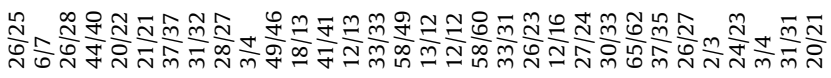

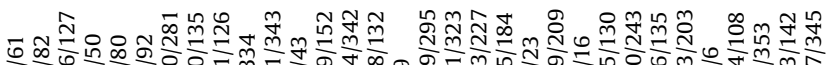

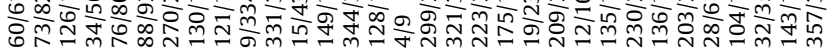

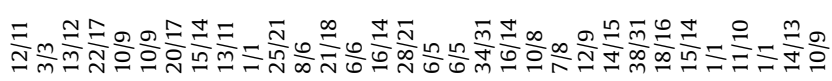

م

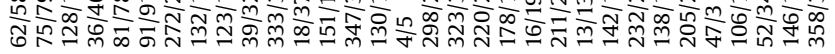

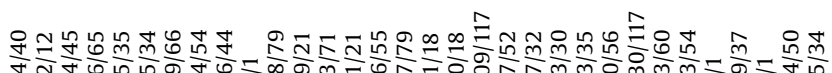

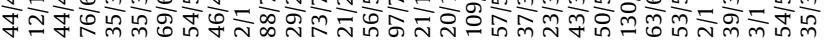

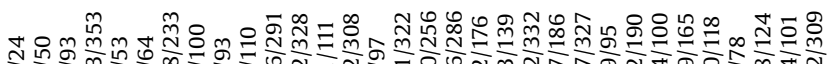

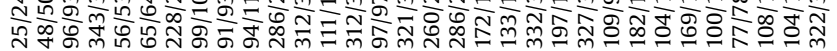

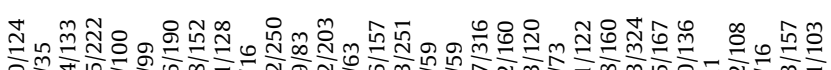

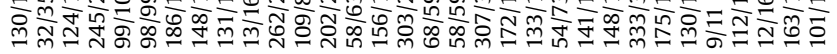

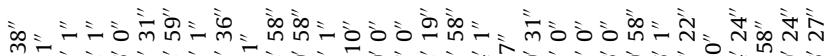

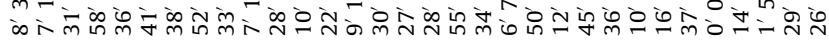

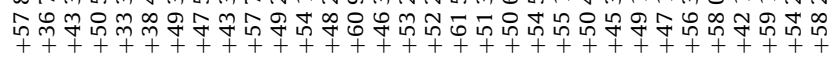

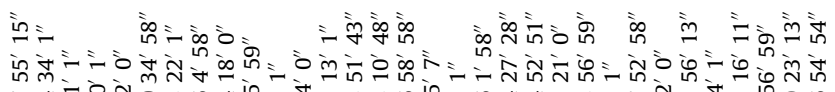

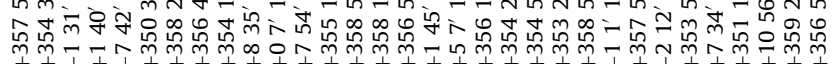

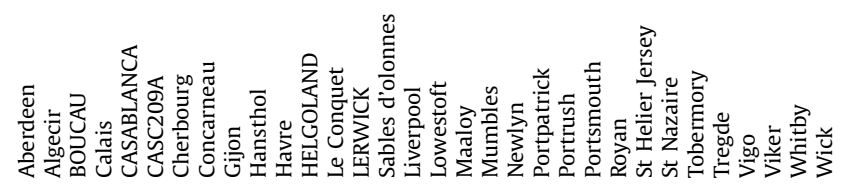




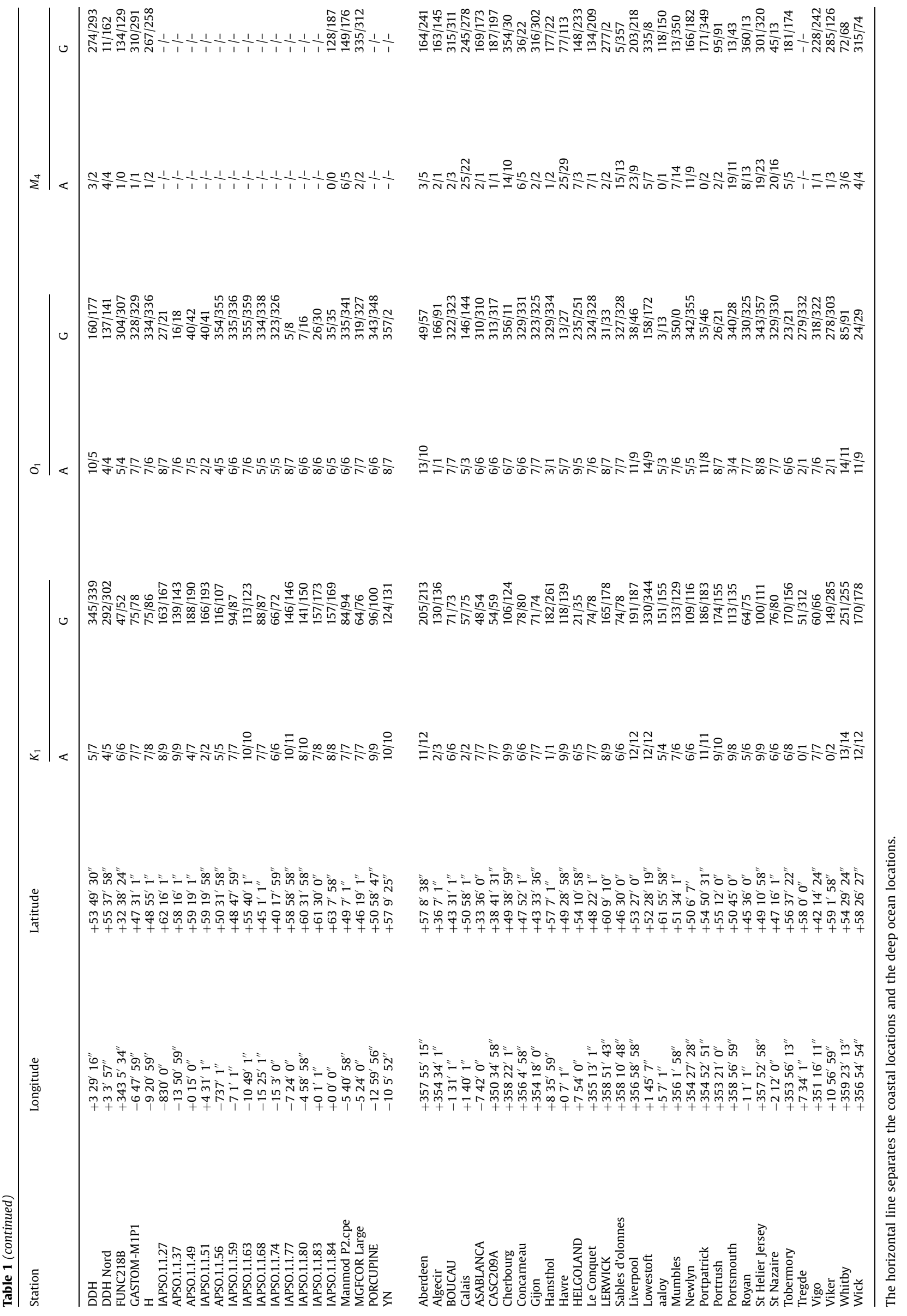


Table 2

Error budget: comparison with the coastal tide gauges data set

\begin{tabular}{|c|c|c|c|c|c|c|c|c|}
\hline \multirow[t]{2}{*}{ Wave } & \multirow[t]{2}{*}{ Model } & \multicolumn{2}{|l|}{$\Delta a$} & \multicolumn{2}{|l|}{$\Delta G$} & \multicolumn{3}{|l|}{$e$} \\
\hline & & Mean & RMS & Mean & RMS & Mean & RMS & RMS* \\
\hline \multirow[t]{3}{*}{$M_{2}$} & NEA-2004 & 4 & 13 & -1 & 8 & 2 & 15 & 15 \\
\hline & FES2004 & 4 & 24 & -1 & 8 & 5 & 26 & 27 \\
\hline & GOT00 & 5 & 23 & 2 & 10 & 3 & 24 & 24 \\
\hline \multirow[t]{3}{*}{$S_{2}$} & NEA-2004 & 3 & 6 & 5 & 16 & 1 & 6 & 6 \\
\hline & FES2004 & 3 & 11 & -5 & 14 & 1 & 12 & 12 \\
\hline & GOT00 & 3 & 11 & 1 & 13 & 1 & 11 & 11 \\
\hline \multirow[t]{3}{*}{$N_{2}$} & NEA-2004 & 1 & 3 & 0 & 7 & 1 & 6 & 6 \\
\hline & FES2004 & 1 & 6 & -1 & 13 & 1 & 6 & 7 \\
\hline & GOT00 & 1 & 5 & 0 & 9 & 1 & 5 & 5 \\
\hline \multirow[t]{3}{*}{$M_{4}$} & NEA-2004 & 0 & 4 & -14 & 63 & 1 & 5 & 5 \\
\hline & FES2004 & 3 & 4 & -39 & 65 & 1 & 7 & 7 \\
\hline & GOT00 & - & - & - & - & - & - & 8 \\
\hline
\end{tabular}

Unit in $\mathrm{cm}$. The GOT00 atlas does not include a $M_{4}$ constituent, the $8 \mathrm{~cm}$ RMS is given with reference to a zero-valued solution, and thus indicates the subsequent additional error when this constituent is omitted in the tidal predictions.

Table 3

Error budget: comparison with the shelf and deep ocean tide gauges data set

\begin{tabular}{|c|c|c|c|c|c|c|c|c|}
\hline \multirow[t]{2}{*}{ Wave } & \multirow[t]{2}{*}{ Model } & \multicolumn{2}{|l|}{$\delta a$} & \multicolumn{2}{|l|}{$\delta G$} & \multicolumn{3}{|l|}{$e$} \\
\hline & & Mean & RMS & Mean & RMS & Mean & RMS & RMS* \\
\hline \multirow[t]{3}{*}{$M_{2}$} & NEA-2004 & 1 & 3 & 0 & 3 & 2 & 4 & 4 \\
\hline & FES2004 & 0 & 2 & 0 & 1 & 0 & 1 & 2 \\
\hline & GOT00 & 0 & 2 & 0 & 1 & 0 & 2 & 2 \\
\hline \multirow[t]{3}{*}{$S_{2}$} & NEA-2004 & 2 & 2 & 3 & 6 & 1 & 3 & 3 \\
\hline & FES2004 & 0 & 1 & 3 & 6 & 1 & 2 & 2 \\
\hline & GOT00 & 0 & 1 & 1 & 5 & 1 & 2 & 2 \\
\hline \multirow[t]{3}{*}{$N_{2}$} & NEA-2004 & 0 & 1 & 1 & 2 & 0 & 1 & 1 \\
\hline & FES2004 & 0 & 1 & 0 & 2 & 0 & 1 & 1 \\
\hline & GOT00 & 0 & 1 & 0 & 4 & 0 & 1 & 1 \\
\hline \multirow[t]{3}{*}{$M_{4}$} & NEA-2004 & 0 & 1 & -24 & 54 & 1 & 2 & 2 \\
\hline & FES2004 & 1 & 1 & -14 & 57 & 1 & 2 & 2 \\
\hline & GOT00 & - & - & - & - & - & - & 2 \\
\hline
\end{tabular}

Unit in $\mathrm{cm}$. Same remark as in Table 2 about GOT00 $M_{4}$ figure.

the pressure (all quantities given per surface unit). The latter is associated with the propagation of tidal energy in the ocean. When considering the mean energy budget integrated over a given domain, this contribution is often transformed so as to emphasize the so-called energy flux. Using Green's formula to transform the pressure gradient RoW, we get

$$
\begin{aligned}
H \mathbf{u} \cdot \nabla \eta & =\nabla \cdot(\eta H \mathbf{u})-\eta \nabla \cdot(H \mathbf{u}) \\
& =\nabla \cdot(\eta H \mathbf{u})+\eta \frac{\partial \eta}{\partial t} \\
& =\nabla \cdot(\eta H \mathbf{u})+\frac{1}{2} \frac{\partial \eta^{2}}{\partial t}
\end{aligned}
$$

When integrating the pressure gradient RoW over a given domain, the divergence term in Eq. (10) is transformed into a flux integral:

$$
-\rho g \int_{\Omega} H \mathbf{u} \cdot \nabla \eta \mathrm{d} s=-\rho g\left(\frac{1}{2} \int_{\Omega} \frac{\partial \eta^{2}}{\partial t} \mathrm{~d} s+\int_{\Gamma} \eta H \mathbf{u} \cdot \mathbf{n} \mathrm{d} l\right)
$$

\begin{tabular}{|c|c|c|c|c|c|c|c|c|}
\hline \multirow[t]{2}{*}{ Wave } & \multirow[t]{2}{*}{ Model } & \multicolumn{2}{|l|}{$\delta a$} & \multicolumn{2}{|l|}{$\delta G$} & \multicolumn{3}{|l|}{$e$} \\
\hline & & Mean & RMS & Mean & RMS & Mean & RMS & RMS* \\
\hline \multirow[t]{3}{*}{$M_{2}$} & NEA-2004 & 0 & 3 & 0 & 10 & 1 & 5 & 5 \\
\hline & FES2004 & 0 & 2 & 1 & 3 & 1 & 2 & 2 \\
\hline & GOT00 & 0 & 1 & 0 & 1 & 0 & 1 & 1 \\
\hline \multirow[t]{3}{*}{$S_{2}$} & NEA-2004 & 0 & 1 & 2 & 9 & 1 & 2 & 2 \\
\hline & FES2004 & -1 & 1 & -1 & 6 & 1 & 2 & 2 \\
\hline & GOT00 & -1 & 1 & -1 & 5 & 0 & 2 & 2 \\
\hline \multirow[t]{3}{*}{$N_{2}$} & NEA-2004 & 0 & 1 & 1 & 5 & 0 & 1 & 1 \\
\hline & FES2004 & 0 & 1 & 1 & 6 & 0 & 1 & 1 \\
\hline & GOT00 & 0 & 1 & 0 & 4 & 0 & 1 & 1 \\
\hline \multirow[t]{3}{*}{$M_{4}$} & NEA-2004 & 0 & 1 & -9 & 81 & 0 & 1 & 1 \\
\hline & FES2004 & 0 & 1 & -21 & 76 & 0 & 1 & 1 \\
\hline & GOT00 & - & - & - & - & - & - & 1 \\
\hline
\end{tabular}

Table 4

Error budget: comparison with the T/P cross-over analysis data set

Unit in $\mathrm{cm}$. Same remark as in Table 2 about GOT00 $M_{4}$ figure.

where $\rho g_{\eta} H \mathbf{u} \cdot \mathbf{n}$ is the so-called energy flux. By averaging in time, it is easy to see that the mean energy flux budget matches the pressure gradient budget:

$$
\begin{aligned}
- & \rho g \lim _{T \rightarrow \infty} \frac{1}{T} \int_{0}^{T} \int_{\Omega} H \mathbf{u} \cdot \nabla \eta \mathrm{d} s \\
= & -\rho g \lim _{T \rightarrow \infty} \frac{1}{T}\left[\int_{\Omega} \eta^{2} \mathrm{~d} s\right]_{0}^{T} \\
& -\rho g \lim _{T \rightarrow \infty} \frac{1}{T} \int_{0}^{T} \int_{\Gamma} \eta H \mathbf{u} \cdot \mathbf{n} \mathrm{d} l \\
= & -\rho g \lim _{T \rightarrow \infty} \frac{1}{T} \int_{0}^{T} \int_{\Gamma} \eta H \mathbf{u} \cdot \mathbf{n} \mathrm{d} l
\end{aligned}
$$

Finally, the time-averaged domain-integrated energy equation can be expressed as follows:

$\lim _{T \rightarrow \infty} \frac{1}{T} \int_{0}^{T} \int_{\Gamma}\left(e_{k}+\rho g \eta H\right) \mathbf{u} \cdot \mathbf{n} \mathrm{d} l=W_{\mathbf{F}}+W_{\mathbf{D}}$

where $W_{\mathbf{F}}=\int_{\Omega} \bar{w}_{\mathbf{F}} \mathrm{d} s$ and $W_{\mathbf{D}}=\int_{\Omega} \bar{w}_{\mathbf{D}} \mathrm{d}$. We can notice that the instantaneous kinetic energy equation (5) can be reformulated as a sort of total energy equation:

$\frac{\partial}{\partial t}\left(e_{k}+e_{p}\right)+\nabla \cdot\left(e_{k} \mathbf{u}\right)+\rho g \nabla \cdot(\eta H \mathbf{u})=\rho H \mathbf{u} \cdot \mathbf{F}+\rho H \mathbf{u} \cdot \mathbf{D}$

where $e_{p}=\frac{1}{2} \rho g \eta^{2}$ is the barotropic potential energy. At any moment, the derivative of the total barotropic energy and the energy divergence are balanced by the RoW of the forces applied to the fluid per surface units. When averaging in time and integrating over a given domain, the mean energy flux at the open boundaries is balanced by the mean RoW of the forces applied to the fluid.

\subsubsection{Spectral energy}

The above equations allow the computation of global energy estimates, not distinguishing between the specific contributions of each tidal constituent (Fig. 7). In fact, because of the nonlinear physics of the tides (due to the divergence transport, advection and nonlinear bottom friction), and of the ensuing wave-to-wave interactions, such a distinction can only be approximative. For example, the $M_{2}$ tide is only the overwhelming part of a physical wave which includes all $M_{2}$ harmonics. Still, it makes sense to attempt to separate the contribution of each constituent when 


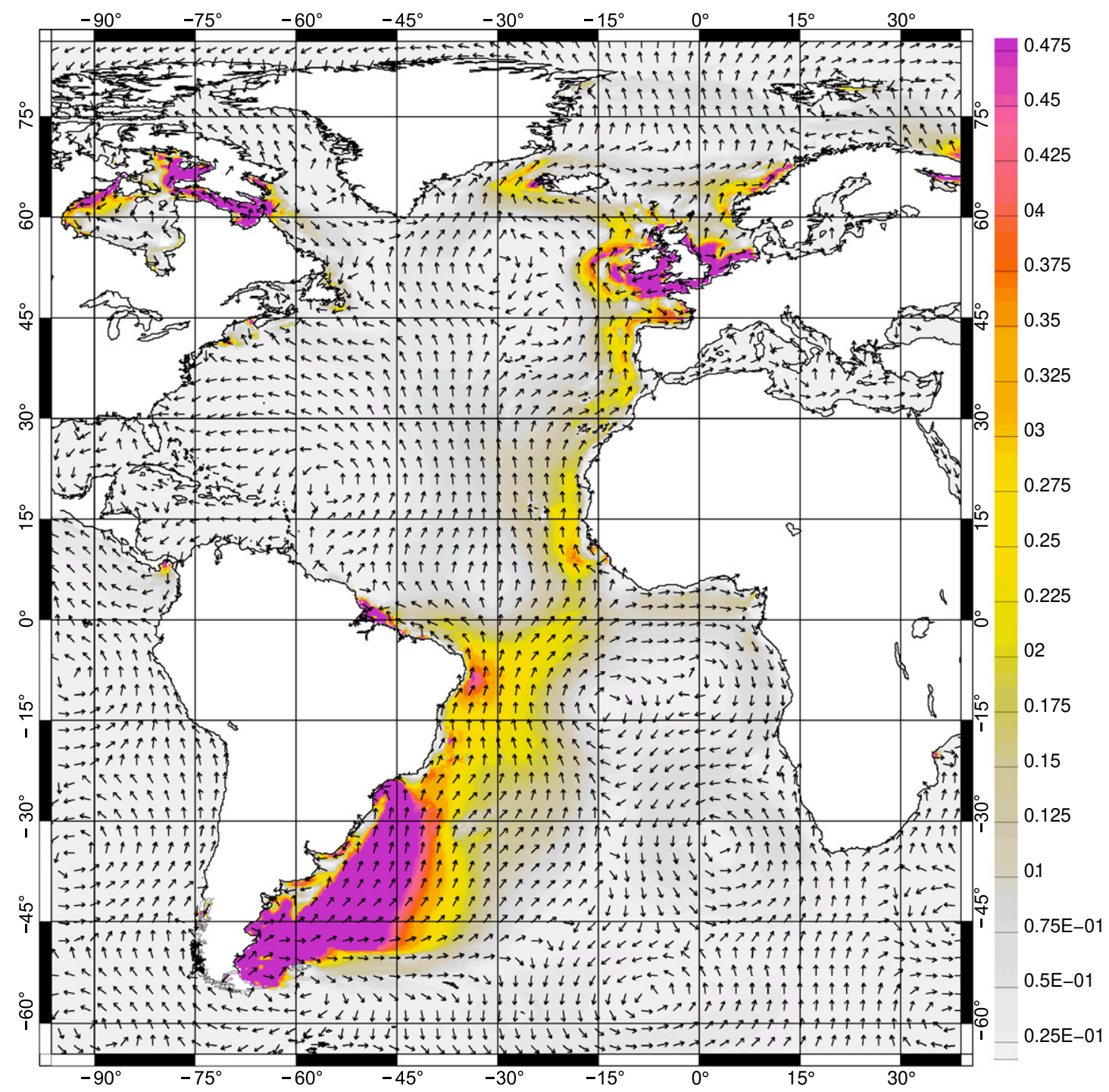

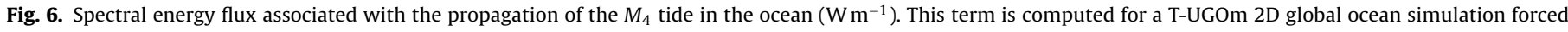

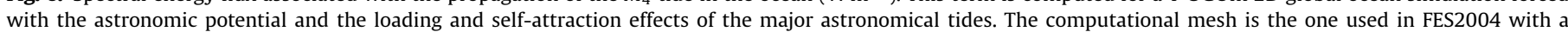
Lagrange-P1 approximation.

considering the mean energy budget. The mean kinetic energy (MKE) per surface unit is defined as

$$
\begin{aligned}
\bar{e}_{k}= & \lim _{T \rightarrow \infty} \frac{1}{T} \int_{0}^{T} e_{k} \mathrm{~d} t=\lim _{T \rightarrow \infty} \rho h \frac{1}{T} \int_{0}^{T}(\mathbf{u} \cdot \mathbf{u}) \mathrm{d} t \\
& +\lim _{T \rightarrow \infty} \rho \frac{1}{T} \int_{0}^{T}(\eta \mathbf{u} \cdot \mathbf{u}) \mathrm{d} t
\end{aligned}
$$

If we assume that the model elevation and barotropic currents are purely harmonic, i.e. that elevation and both velocity components take the following form:

$\alpha(\lambda, \phi, t)=\sum_{i} \hat{\alpha}_{i}(\lambda, \phi) \cos \left(\omega_{i} t+V_{0}-G_{\alpha, i}(\lambda, \phi)\right)$

where the subscript $i$ stands for the tidal component index, then, owing to the orthogonality of the harmonic functions, Eq. (15) can be reduced to

$\bar{e}_{k}=\sum_{i} \lim _{T \rightarrow \infty} \rho h \frac{1}{T} \int_{0}^{T}\left(\mathbf{u}_{i} \cdot \mathbf{u}_{i}\right) \mathrm{d} t+\sum_{i, j, l} \lim _{T \rightarrow \infty} \rho \frac{1}{T} \int_{0}^{T}\left(\eta_{i} \mathbf{u}_{j} \cdot \mathbf{u}_{l}\right) \mathrm{d} t$
Nonzero terms in the second summation on the righthand side are those that satisfy $\omega_{i} \pm \omega_{j} \pm \omega_{k}=0$. To first order (i.e. replacing the total depth $H$ by the mean depth $h$ ), the mean kinetic energy simplifies in

$$
\begin{aligned}
\bar{e}_{k} & \simeq \lim _{T \rightarrow \infty} \rho h \frac{1}{T} \int_{0}^{T}(\mathbf{u} \cdot \mathbf{u}) \mathrm{d} t \\
& =\rho h \sum_{i} \frac{1}{T_{i}} \int_{0}^{T_{i}}\left(\mathbf{u}_{i} \cdot \mathbf{u}_{i}\right) \mathrm{d} t \\
& =\rho h \frac{1}{4} \sum_{i}\left(\hat{\mathbf{u}}_{i} \cdot \hat{\mathbf{u}}_{i}\right)
\end{aligned}
$$

where $\hat{\mathbf{u}}_{i}$ is the amplitude of the complex velocity.

Accordingly, the total MKE can be approximated by the summation of the MKEs specific to each tidal constituent. Similar considerations can be applied to the various contributions to the mean energy budget. Multiplying Eq. (1) by $\rho g h \mathbf{u}_{i}$ and averaging 
a
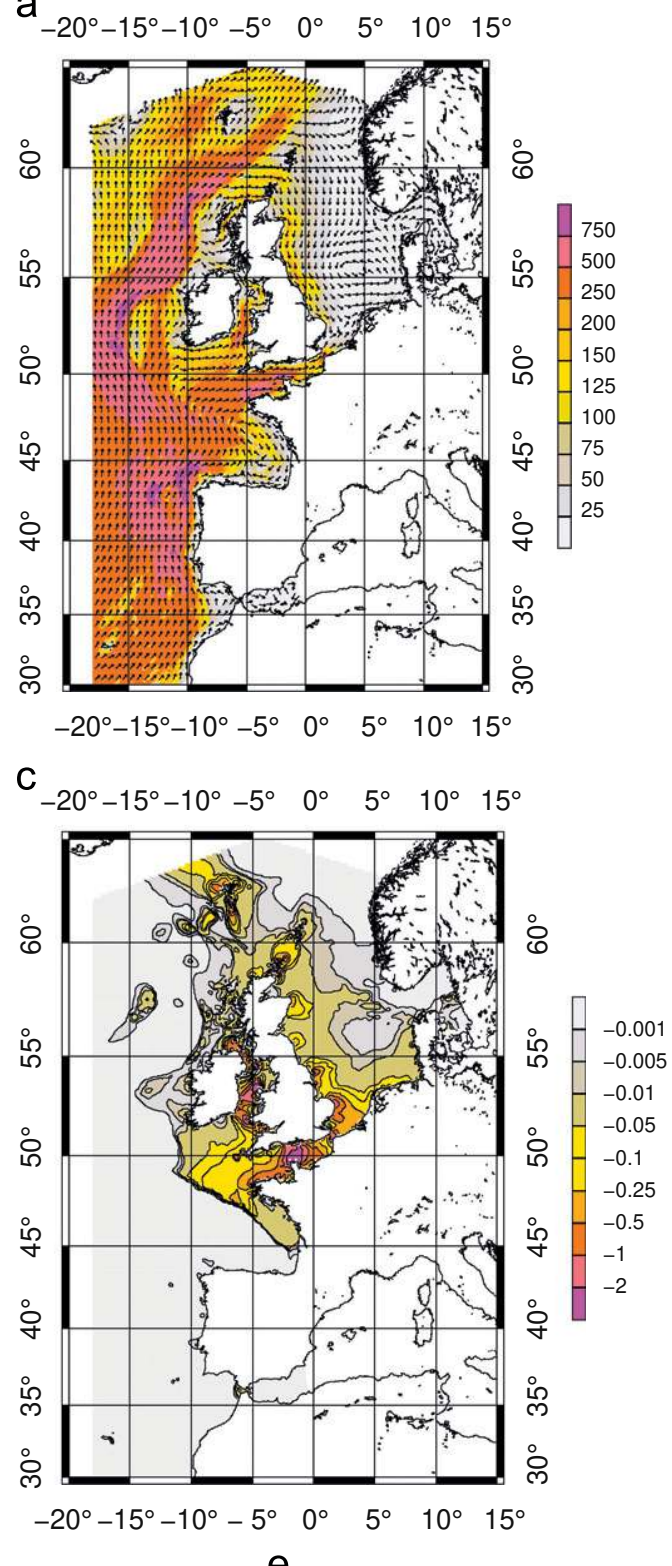

b
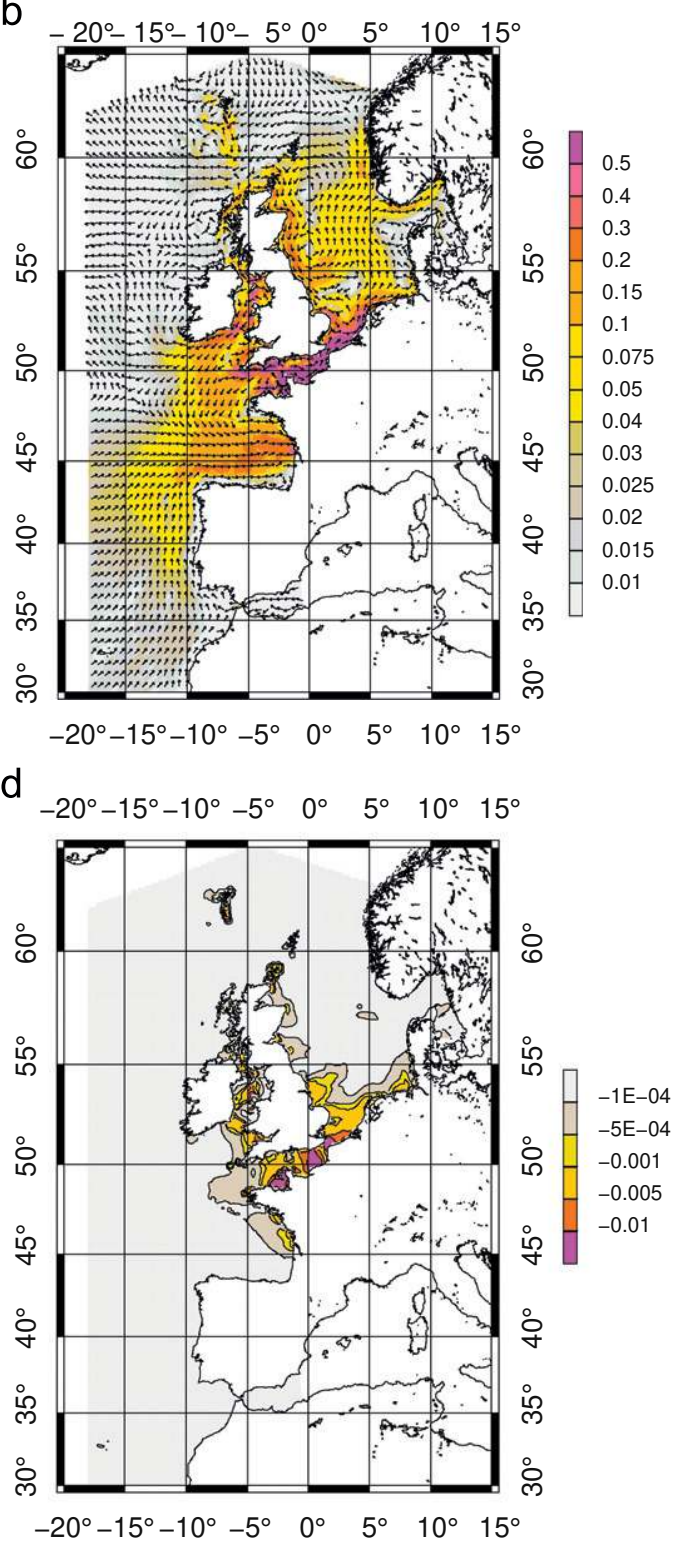

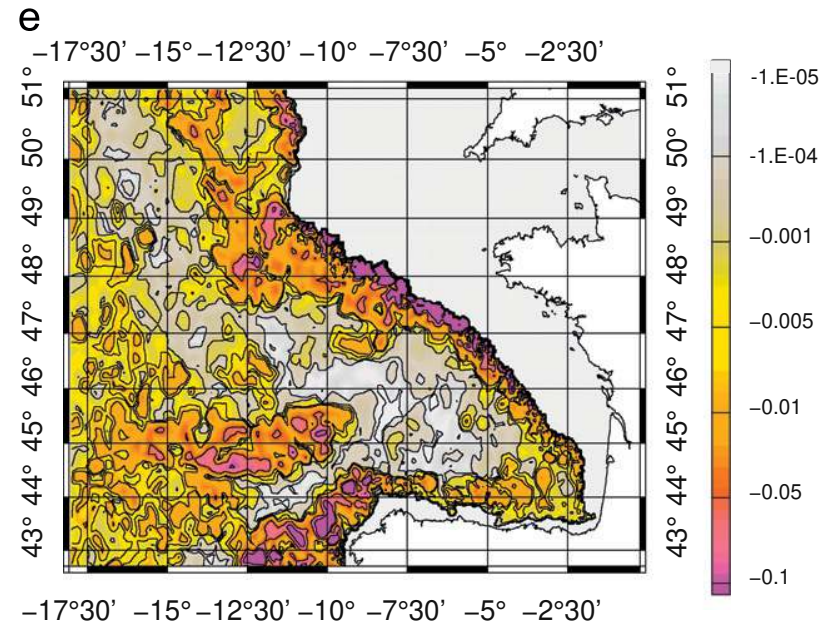

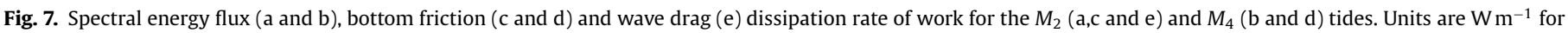
the fluxes and $\mathrm{Wm}^{-2}$ for the rate of work. 
Table 5

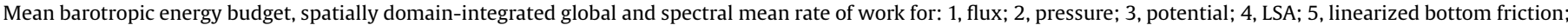
6 , full bottom friction; 7 , viscosity; 8 , wave drag; 9, energy advection

\begin{tabular}{|c|c|c|c|c|c|c|c|c|c|c|}
\hline Wave & Model & 1 & 2 & 3 & 4 & 5 & 6 & 7 & 8 & 9 \\
\hline \multirow[t]{3}{*}{ Global } & NEA-2004 & - & 300.0 & -0.6 & -20.0 & - & -221.0 & -14.0 & -44.0 & 0.0 \\
\hline & NEA-2004 & 265 & 248.5 & -0.5 & -16.5 & -175.0 & -182.5 & -10.0 & -36.5 & 1.5 \\
\hline & FES99 & - & - & - & - & -270 & - & - & - & - \\
\hline \multirow[t]{2}{*}{$M_{2}$} & Miller & 180 & - & - & - & - & - & - & - & - \\
\hline & Cartwright et al. & 360 & - & - & - & - & - & - & - & - \\
\hline$S_{2}$ & NEA-2004 & 36.5 & 34.5 & -1.0 & -2.5 & -23.0 & -25.0 & -1.5 & -4.5 & 0.0 \\
\hline$K_{1}$ & NEA-2004 & 2.8 & 1.9 & 0.3 & -0.2 & -0.8 & -1.0 & -0.1 & -0.8 & 0.0 \\
\hline$M_{4}$ & NEA-2004 & 0.0 & 0.7 & 0.0 & 0.0 & -0.8 & -0.9 & -0.1 & 0.0 & -0.3 \\
\hline
\end{tabular}

Unit in GW.

in time lead to the spectral energy equation:

$\rho h \lim _{T \rightarrow \infty} \frac{1}{T} \int_{0}^{T}(\mathbf{u} \cdot \nabla \mathbf{u}) \cdot \mathbf{u}_{i} \mathrm{~d} t=\rho h \frac{1}{T_{i}} \int_{0}^{T_{i}}(\mathbf{u} \cdot \nabla \mathbf{u})_{i} \cdot \mathbf{u}_{i} \mathrm{~d} t=\bar{w}_{a, i}$

Applying a similar treatment to all terms in Eq. (1) yields the time-averaged spectral energy equation:

$\bar{w}_{a, i}=\bar{w}_{p, i}+\bar{w}_{\mathbf{F}, i}+\bar{w}_{\mathbf{D}, i}$

where

$\bar{w}_{p, i}=-\rho g h \frac{1}{T_{i}} \int_{0}^{T_{i}} \mathbf{u}_{i} \cdot \nabla \eta_{i} \mathrm{~d} t$

$\bar{w}_{\mathbf{F}, i}=\rho h \frac{1}{T_{i}} \int_{0}^{T_{i}} \mathbf{u}_{i} \cdot \mathbf{F}_{i} \mathrm{~d} t$

$\bar{w}_{\mathbf{D}, i}=\rho h \frac{1}{T_{i}} \int_{0}^{T_{i}} \mathbf{u}_{i} \cdot \mathbf{D}_{i} \mathrm{~d} t$

Expanding further, we obtain

$\bar{w}_{\mathbf{F}, i}=\bar{w}_{a s t, i}+\bar{w}_{L S A, i}=\rho g h \frac{1}{T_{i}} \int_{0}^{T_{i}} \mathbf{u}_{i} \cdot \nabla \Pi_{i} \mathrm{~d} t$

$\bar{w}_{\mathbf{D}, i}=\bar{w}_{w, i}+\bar{w}_{d, i}+\bar{w}_{b, i}$

where

$\bar{w}_{w, i}=-\rho \operatorname{ch} \frac{1}{T_{i}} \int_{0}^{T_{i}}\left(\nabla h \cdot \mathbf{u}_{i}\right)^{2} \mathrm{~d} t$

$\bar{w}_{d, i}=\rho h \frac{1}{T_{i}} \int_{0}^{T_{i}} \mathbf{u}_{i} \cdot(\nabla \cdot(A \nabla \mathbf{u}))_{i} \mathrm{~d} t$

$\bar{w}_{b, i}=-\rho C h \frac{1}{T_{i}} \int_{0}^{T_{i}} \mathbf{u}_{i} \cdot\left(\frac{\|\mathbf{u}\| \mathbf{u}}{H}\right)_{i} \mathrm{~d} t$

Here $\bar{w}_{p, i}, \bar{w}_{\mathbf{F}, i}, \bar{w}_{w, i}, \bar{w}_{d, i}$ and $\bar{w}_{b, i}$ are the surface pressure, tidal forcing, wave drag, horizontal diffusivity and bottom friction contributions. The computation, for each harmonic component, of their spectral RoW per surface unit is straightforward from their tidal constants, for the linear terms in Eq. (1). The same is true of the surface pressure gradient, tidal forcing and wave drag. The same is not true, however, of the bottom friction and horizontal momentum diffusivity; for the latter, the nonlinearities come from the Smagorinsky scheme used to establish the diffusion coefficient. The quantities in Eqs. (24) and (25) can be computed by harmonic analysis of the terms $(\nabla \cdot(A \nabla \mathbf{u}))$ and $(\|\mathbf{u}\| \mathbf{u})$ at simulation time. It can also be analyzed off-line by using a tidal current prediction (actually this method was used to produce the estimates shown in this paper). For the bottom friction, a more efficient procedure consists in using the quasi-linearization technique of the bottom friction coefficient (Le Provost et al., 1981). This approach is used in the CEFMO spectral numerical model (Le Provost et al., 1994), from which the (prior) hydrodynamic tidal solutions were derived to produce the FES assimilated tidal atlases. It requires the assumption that the currents of a given dominant constituent are significantly larger than those of any other tidal constituent. This assumption, called the dominant wave assumption, is generally reasonable in regions where friction is significant, i.e. in the shelf seas. The amplitude of the tides in these regions is controlled by a local resonance dependent on the shelf geometry, and such resonance will affect the different tidal species differently. In other words, either diurnal or semi-diurnal tides will be greatly amplified, but rarely both together. In such conditions, the existence of a dominant wave is generally observed, mostly $M_{2}$. Based on this assumption, and by applying a perturbation method in complex space, the friction coefficients are linearized; one set is related to the dominant wave, and the other set is related to all the other tidal constituents together. In the Bay of Biscay, $M_{2}$ is clearly the dominant wave, and its tidal friction can be approximated by the following expression:

$\tilde{\alpha}_{i}=\hat{\alpha}_{i} \mathrm{e}^{-\mathrm{j} G_{\alpha, i}}$

where $\mathrm{j}=\sqrt{-1}$ and

$\left(\frac{\| \widetilde{\mathbf{u} \| \mathbf{u}}}{H}\right)_{M_{2}} \sim \frac{1}{h}\left(\begin{array}{cc}r & r^{\prime} \\ r^{\prime \prime} & r^{\prime \prime \prime}\end{array}\right) \tilde{\mathbf{u}}_{M_{2}}$

where the complex coefficients are defined as

$r=r^{\prime \prime \prime}=\frac{C}{h} R_{1}, \quad r^{\prime}=r^{\prime \prime}=-\frac{C}{h} \mathrm{j} R_{1}^{\prime}$

For any other tidal component, the tidal friction can be approximated by a similar expression, of coefficients:

$r=\frac{C}{h}\left(R_{k}+R_{k}^{\prime}\right), \quad r^{\prime}=r^{\prime \prime}=\frac{C}{h} R_{k}^{\prime \prime}$

$r^{\prime \prime \prime}=\frac{C}{h}\left(R_{k}-R_{k}^{\prime}\right)$

The coefficients $R_{1}, R_{1}^{\prime}, R_{k}, R_{k}^{\prime}$ and $R_{k}^{\prime \prime}$ are real and depend only on the dominant wave currents, $M_{2}$ in our application. Their computation is straightforward and cost-effective, and the loss in accuracy when computing the RoW of the bottom friction does not usually exceed $5-10 \%$. This loss is regarded as reasonable, especially compared to the other uncertainties of the model. It shows that the linearization technique can be used efficiently when linearized equations are needed (for instance to derive adequate spectral barotropic tidal currents from tidal elevation at the open boundaries of the model). The space integral of the mean RoW per surface unit leads to the energy budget over the model domain.

As a final step, the spectral mean energy flux of a given tidal constituent is defined by the usual expression:

$\bar{\Phi}_{i}=\rho g \frac{1}{T_{i}} \int_{0}^{T_{i}} \int_{\Gamma} \eta_{i} h \mathbf{u}_{i} \cdot \mathbf{n} \mathrm{d} l$ 
The mean energy flux can be written as the sum of the spectral mean energy fluxes plus cross terms as follows:

$$
\begin{aligned}
\bar{\Phi} & =\rho g \lim _{T \rightarrow \infty} \frac{1}{T} \int_{0}^{T} \int_{\Gamma} \eta H \mathbf{u} \cdot \mathbf{n} \mathrm{d} l \\
& =\sum_{i} \bar{\Phi}_{i}+\rho g \sum_{i, j, k} \lim _{T \rightarrow \infty} \frac{1}{T} \int_{0}^{T} \int_{\Gamma} \eta_{i} \eta_{j} \mathbf{u}_{k} \cdot \mathbf{n} \mathrm{d} l
\end{aligned}
$$

Nonzero terms in the second summation at the righthand side are those that satisfy $\omega_{i} \pm \omega_{j} \pm \omega_{k}=0$. This summation involves triple wave interactions (hence at least one order smaller or more than the double wave interactions) and also major triplets, i.e. triplets including a major tidal constituent, and will necessarily include a nonlinear or low-frequency constituent which magnitude is extremely small in the deep ocean (such as $M_{2} \times M_{2} \times M_{4}$ ). As a consequence, this summation can be neglected in our case where the open ocean boundaries are located in the deep ocean. Then the total mean energy flux can be approximated by the summation of the spectral energy fluxes.

Unfortunately, when using the linearized energy budget, Eq. (11) is no longer verified and Eq. (30) is no longer consistent with the estimate of the pressure gradient RoW. As reported in Table 5 , the inconsistency reaches less than $10 \%$ of the total RoW for the $M_{2}$ constituent. To understand it, let us start from the core of the RoW of pressure forces:

$$
\begin{aligned}
H \mathbf{u} \cdot \nabla \eta & =h \mathbf{u} \cdot \nabla \eta+\eta \mathbf{u} \cdot \nabla \eta \\
& =h \mathbf{u} \cdot \nabla \eta+\nabla \cdot\left(\eta^{2} \mathbf{u}\right)-\rho g \eta \nabla \cdot(\eta \mathbf{u})
\end{aligned}
$$

Substituting Eq. (32) in Eq. (10), we get

$\nabla \cdot(\eta h \mathbf{u})+\frac{1}{2} \frac{\partial \eta^{2}}{\partial t}=h \mathbf{u} \cdot \nabla \eta-\rho g \eta \nabla \cdot(\eta \mathbf{u})$

Integrating over the domain and averaging in time, we obtain

$$
\begin{aligned}
\sum_{i} \bar{\Phi}_{i} & =-\sum_{i} \bar{w}_{p, i}-\rho g \lim _{T \rightarrow \infty} \frac{1}{T} \int_{0}^{T} \int_{\Omega} \eta \nabla \cdot(\eta \mathbf{u}) \mathrm{d} s \mathrm{~d} t \\
& =-\sum_{i} \bar{w}_{p, i}-\rho g \sum_{i, j, k} \lim _{T \rightarrow \infty} \frac{1}{T} \int_{0}^{T} \int_{\Omega} \eta_{i} \nabla \cdot\left(\eta_{j} \mathbf{u}_{k}\right) \mathrm{d} s \mathrm{~d} t
\end{aligned}
$$

Again, nonzero terms in the second summation on the righthand side are those that satisfy $\omega_{i} \pm \omega_{j} \pm \omega_{k}=0$. They represent the discrepancy between the spectral estimate of the pressure RoW and the spectral mean energy flux. Since the sum of the spectral mean energy fluxes is close to the total mean energy flux, and hence to the mean RoW of pressure forces, it quantifies the limit of the spectral approach for the tidal energy estimation. This point deserves mention, as the mean spectral energy flux is used by some authors to infer the mean spectral RoW of the pressure forces in regions of interest, in order to estimate the local balance between tidal generation and dissipation. In the case of astronomical tides, the generation term can be considered as negligible, and the energy fluxes are used as a dissipation estimate. The omission of the coupled terms is of small importance for the main tidal constituents, but conversely it may be significant for the minor tidal constituents.

From the global and spectral energy budget (Table 5), it can be seen that $M_{2}$ represents more than $80 \%$ of the total energy in the European shelf seas. The figure of $250 \mathrm{GW}$ budget in this region is more or less within the range of previous estimates. It is also to be compared with the $2.45 \mathrm{TW}$ energy budget for the global ocean. The wave drag dissipation is about $18 \%$ of the total dissipation (i.e. wave drag plus bottom friction) instead of $25-30 \%$ on average in the global ocean. The energy figures decrease rapidly with $S_{2}$ and even further with $K_{1}$. The energy involved in $M_{4}$ dynamics is only $1 \mathrm{GW}$, which is very small indeed but still larger than any other nonlinear tidal budget. As mentioned in the previous section, the
Table 6

Characteristics of principal tidal constituents considered in our study

\begin{tabular}{lllllll}
\hline Tidal species & Name & $T(\mathrm{~h})$ & $a(\mathrm{~m})$ & $f$ & $u(\mathrm{rad})$ & $V_{0}(\mathrm{rad})$ \\
\hline Semi-diurnal & & & & & & \\
Principal lunar & $M_{2}$ & 12.421 & 0.242334 & 1.024 & 0.0283441 & 2.57347 \\
Principal solar & $S_{2}$ & 12.000 & 0.112841 & 1 & 0 & 0 \\
Lunar elliptic & $N_{2}$ & 12.658 & 0.046398 & 1.024 & 0.0283441 & 5.49138 \\
Lunisolar & $K_{2}$ & 11.967 & 0.030704 & 0.835 & 0.130586 & 3.82885 \\
& & & & & & \\
Diurnal & & & & & & \\
Lunisolar & $K_{1}$ & 23.935 & 0.141565 & 0.934 & 0.1305859 & 3.82885 \\
Principal lunar & $O_{1}$ & 25.819 & 0.100514 & 0.892 & 6.11445 & 5.02782 \\
Principal solar & $P_{1}$ & 24.066 & 0.046843 & 1 & 0 & 2.45433 \\
Elliptic lunar & $Q_{1}$ & 26.868 & 0.019256 & 0.892 & 6.11445 & 1.66255 \\
\hline
\end{tabular}

The period of the tide is $T, a$ is the equilibrium amplitude for the tide, $u$ and $f$ are the nodal factors (corrective terms, respectively, for the amplitude and phase) and $V_{0}$ is the phase computed for $t_{0}$ taken at the origin time in our modeling, that is $1 \mathrm{st}$ of August 1994.

tidal amplitude is damped excessively in NEA-2004. Further adjustments of the parameters are probably necessary (regarding in particular the wave drag and bottom friction coefficients), which will impact the energy budget.

\section{Barotropic tides in the Bay of Biscay}

As a first step toward the modeling of internal tides of the Bay of Biscay, the 3D coastal ocean model SYMPHONIE is embedded inside the T-UGOm model. The initial solution and the forcing solution imposed at the boundaries in the SYMPHONIE modeling are provided by T-UGOm (velocities and surface elevations). Some comparisons between model solutions and observations are shown in order to validate the surface forcing of internal tides.

\subsection{The SYMPHONIE model configuration}

The SYMPHONIE 3D coastal ocean model is based on primitive equations and classical simplifications, principally the Boussinesq and hydrostatic pressure assumptions (Marsaleix et al., 2008). The model is used in a 2D barotropic shallow-water mode, in order to analyze the tides over two months and a half. The components of the current and the sea surface elevation are computed on a $C$ grid using a classical finite-difference method (Arakawa and Suarez, 1983). High-frequency barotropic processes and fast moving surface waves are explicitly solved with a strong CFL constraint on the time step. Bottom friction is computed through a logarithmic parameterization of the bottom boundary layer. Viscosity coefficients related to the momentum horizontal fluxes are taken to be constant in time but dependent on the size of horizontal grid meshes (namely $15 \mathrm{~m}^{2} \mathrm{~s}^{-1}$ for a horizontal grid mesh of $1.5 \mathrm{~km}$ ) following the formulation by Smagorinsky (1993).

\subsubsection{Model forcing}

Special attention has been paid to the OBC since spurious reflections of outgoing waves at open boundaries can have a significant and negative impact on the inner domain solution. The order of magnitude of time scales associated with the propagation of waves through the Bay of Biscay area (about $1.1 \mathrm{~h}$ for a barotropic semi-diurnal wave) is much shorter than the duration of our simulations. The choice of $\mathrm{OBC}$ has led to the use of the radiative boundary conditions by Flather (1976) for the tidal external field i.e.:

$\left(\eta-\eta_{\text {tide }}\right)=\sqrt{\frac{h+\eta}{g}}\left(\overline{\mathbf{u}}-\overline{\mathbf{u}}_{\text {tide }}\right) \cdot \mathbf{n}$ 


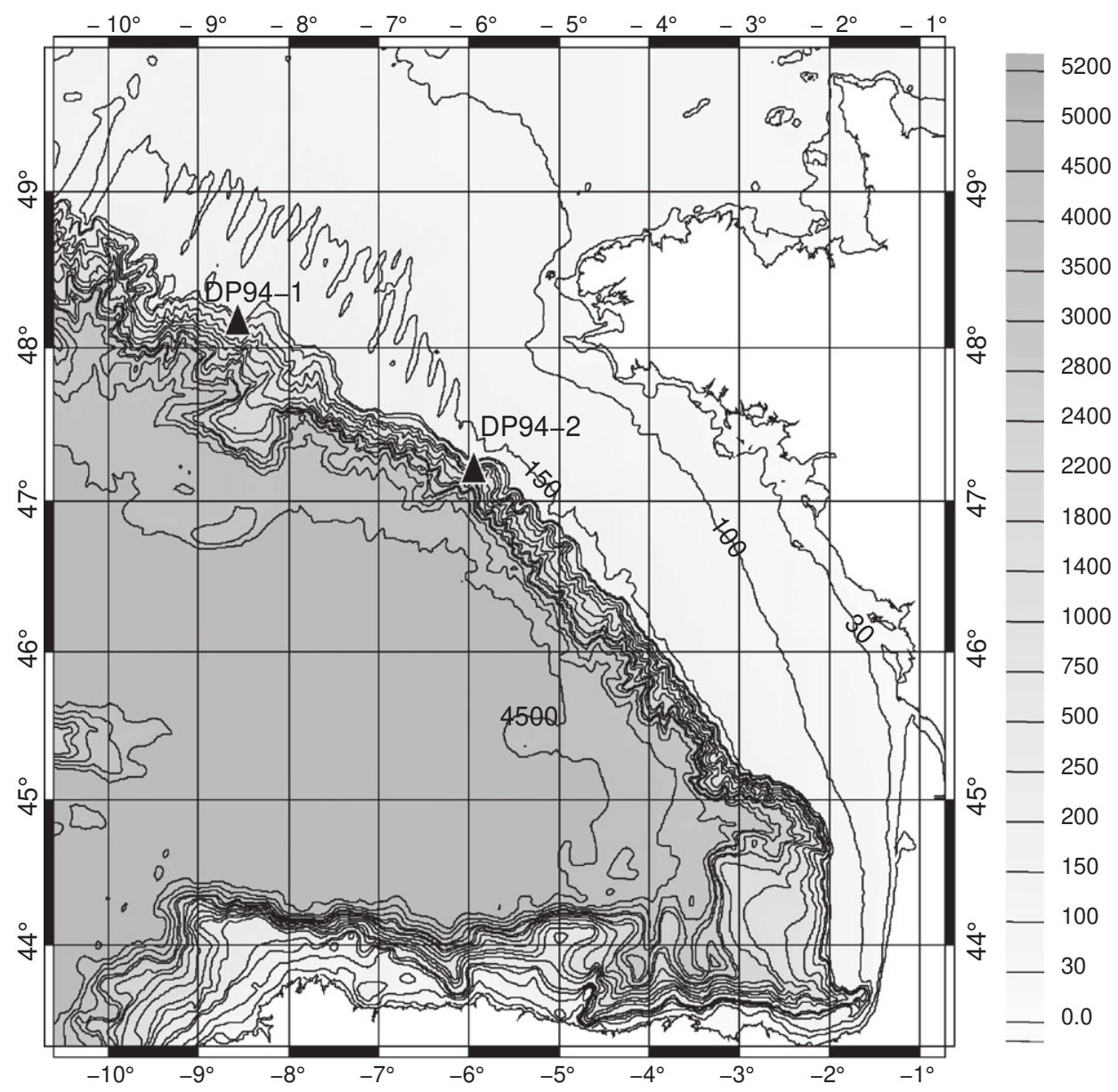

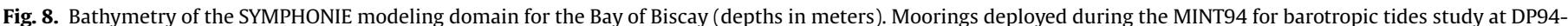
$1(48.11 \mathrm{~N}, 8.49 \mathrm{~W})$ and DP94-2 (47.24N, 5.94W) locations are indicated as white triangles.

where $\eta$ and $\eta_{\text {tide }}$ are the computed sea surface elevation and its external forcing counterpart, respectively, $\overline{\mathbf{u}}$ and $\overline{\mathbf{u}}_{\text {tide }}$ the computed depth-averaged current and its external forcing counterpart, respectively, and $\mathbf{n}$ a unit outward vector normal to the open boundary. Details on the numerical implementation of the $\mathrm{OBC}$ are given in Marsaleix et al. (2006).

Barotropic tidal forcing: $\mathrm{OBC}$ have also the purpose of forcing the inner solution with external fields, which in our case are those of the NEA-2004 atlas. The major tidal constituents for the Bay of Biscay are considered. With regard to the Rayleigh criterion (Pugh, 1989) or the synoptic period, over which two tidal constituents are separated by a cycle, the duration of the recorded or modeled time series necessary to separate two frequencies $f_{1}$ and $f_{2}$ must be of $\tau \geqslant 1 /\left|f_{1}-f_{2}\right|$. Accordingly, the present study may not separate all the tidal components as the separation of $S_{2}$ and $K_{2}$ requires 182 days, even if Davies and Kwong (2000) have shown that the error with a 60 days run was comparable with the error in the current meter data $\left( \pm 2 \mathrm{~cm} \mathrm{~s}^{-1}\right)$ in this case. The forcing only includes tidal elevations and barotropic currents for five constituents: $M_{2}, S_{2}, N_{2}, K_{1}$ and $M_{4}$ (see Appendix A and Table 6). In addition, tidal forcing includes the tidal potential due to astronomical effects, LSA effects (Appendix A).

Modeling domain: The Bay of Biscay is located in the North-East Atlantic ocean $(20-50 \mathrm{~N})$. It is made of a wide continental shelf (with depths lower than $200 \mathrm{~m}$ ) separated from the abyssal plain (with depths reaching more than $4500 \mathrm{~m}$ ) by an abrupt slope. The modeling domain extends from the Iberic peninsula to the English Channel entrance and covers more than $700 \mathrm{~km}$ from East to West and from North to South (Fig. 8).

\subsection{Description of the major tidal component $M_{2}$ and its first harmonic $M_{4}$}

The amplitudes and phase lags of the surface elevation and current ellipses are plotted in Fig. 9 for $M_{2}$ and $M_{4}$. Tidal amplitudes are maximum near the coasts and in the Mont Saint-Michel bay (Figs. 9(a) and (d)) and currents are maximum over the shelf (Figs. 9(c) and (f)).

\subsubsection{The semi-diurnal $M_{2}$ tide}

The $M_{2}$ surface elevation amplitude (Fig. 9(a)) goes up from $1 \mathrm{~m}$ above the abyssal plain to $1.7 \mathrm{~m}$ at the Atlantic coasts with a fast increase on the shelf. Moreover it reaches $4.3 \mathrm{~m}$ in the Mont Saint-Michel bay. This last increase is due to a resonance phenomenon following Clarke (1991), as the shelf width is approximately $135 \mathrm{~km}$ and nearly equal to one quarter of the wavelength $\lambda / 4 \quad(\lambda=c T=\sqrt{g H} T=541.5 \mathrm{~km}$ for a depth of $H=15 \mathrm{~m}$ ). Interferences arise between the waves going in and out of the bay: semi-diurnal waves are Poincare stationnary waves in the Mont Saint-Michel bay (cotidal lines are parallel to the coast in Fig. 9(b)). Maximum tidal amplitudes are observed during the equinox spring tide when it can reach $14 \mathrm{~m}$.

The map of the cotidal lines (Fig. 9(b)) indicates that phases are increasing Northward going from $90^{\circ}$ in the Bay of Biscay to $280^{\circ}$ in the English Channel entrance where the cotidal lines become closer to one another. The Northward propagation in the Bay of Biscay is affected by an acceleration toward the English Channel where the waves propagate Eastward. At the English Channel 
a

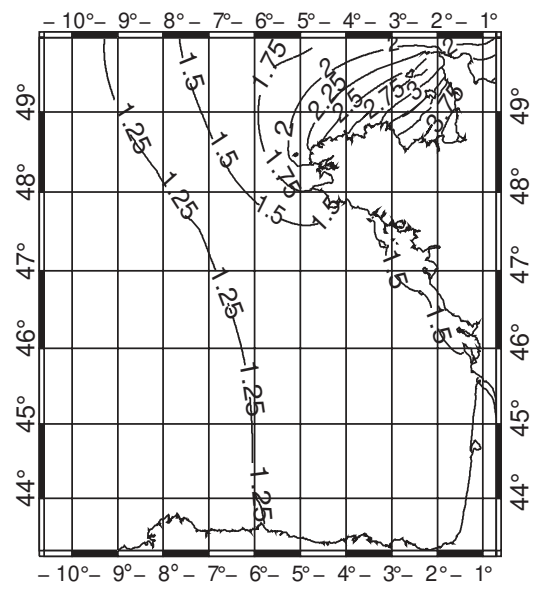

d

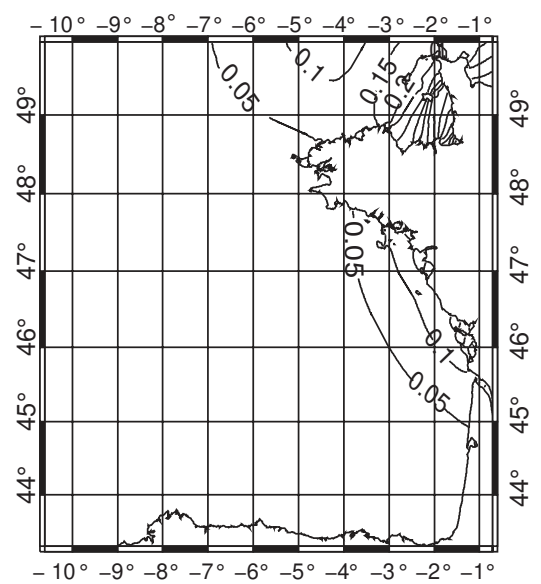

b

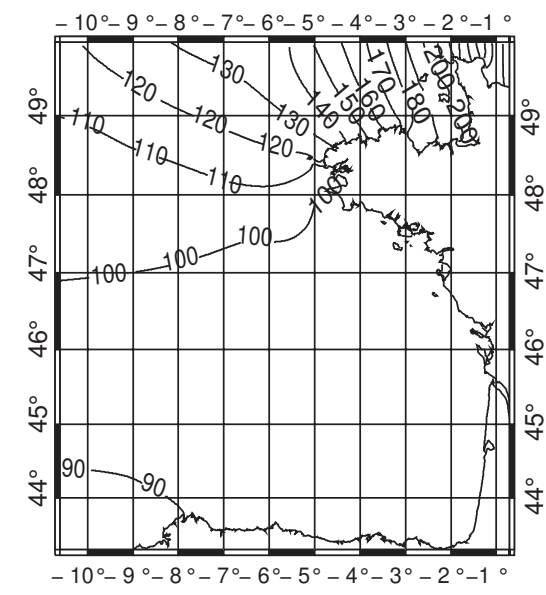

e

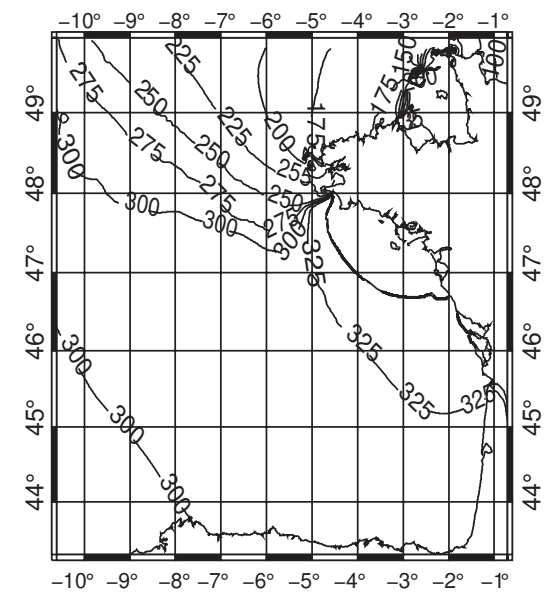

C

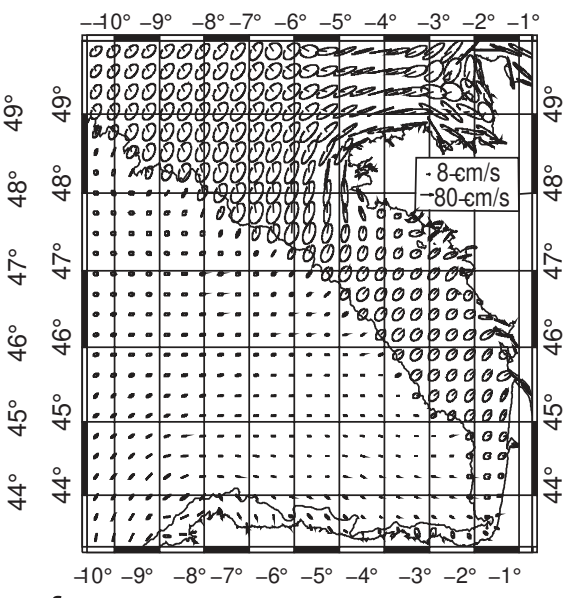

f

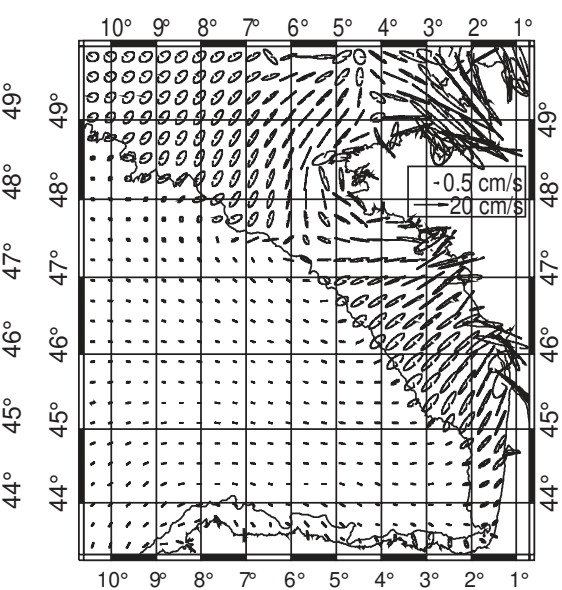

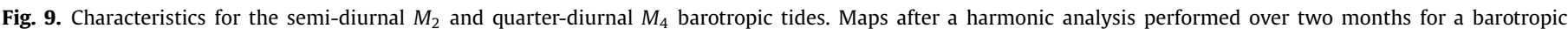

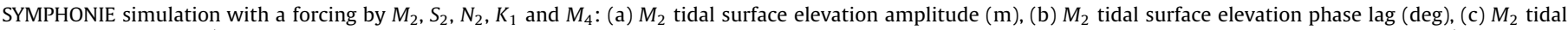
current ellipses $\left(\mathrm{cm} \mathrm{s}^{-1}\right.$ ), (d) $M_{4}$ tidal surface elevation amplitude (m), (e) $M_{4}$ tidal surface elevation phase lag (deg) and (f) $M_{4}$ tidal current ellipses (cm s ${ }^{-1}$ ).

entrance the tide propagates mostly as progressive Kelvin waves parallel to the coast (Fang et al., 1991). Over the abyssal plain waves are propagating as Kelvin waves in the Bay of Biscay (Le Cann, 1990). As far as the continental shelf is concerned, two kinds of waves can be found following the results by Le Cann (1990) and Jezequel and Maze (2001) based on the theory developed by Clarke and Battisti $(1981,1982 a, b)$. As the shelf break is crossed, a Poincaré wave is generated and tidal elevations become in quadrature with tidal currents. The orientation of $M_{2}$ tidal current ellipses (Fig. 9(c)) changes and the influence of a Poincaré wave dominates. On the contrary, closer to the coast the Kelvin character can be dominant again.

From Fig. 9(c) the current ellipses are oriented toward the shelf break above the plain with values ranging from 1 to $5 \mathrm{~cm} \mathrm{~s}^{-1}$ at the Southern boundary. As time elapses the current turns to a cyclonic direction describing an ellipse. Ellipses are nearly circular over the plain; when the shelf break is crossed they become more rectilinear and aligned with the English Channel entrance. Moreover, at the crossing the polarization of the ellipse changes and currents increase rapidly. Above the continental slope the currents are nearly perpendicular to the slope, that is perpendicular to large bathymetry gradients, a favorable condition for internal tides generation (Baines, 1982). Over the shelf, the amplitude of the current is about $20 \mathrm{~cm} \mathrm{~s}^{-1}$ with local accelerations at the coast where the amplitude can be as large as $1 \mathrm{~ms}^{-1}$ (in the Gironde estuary for example). The highest values for the currents are reached in the English Channel entrance and in the Mont SaintMichel bay, with amplitudes of $1.5 \mathrm{~m} \mathrm{~s}^{-1}$. Ellipses can be distorted near the coast under the influence of friction.

\subsubsection{The quarter-diurnal $M_{4}$ harmonic}

The quarter-diurnal component of the tide (Figs. 9(d)-(f)) is generated by nonlinearity from $M_{2}$. It is therefore not surprising that the amplitudes in Fig. 9(d) are larger near the coast than over the plain, as is the case for $M_{2}$. They reach $25 \mathrm{~cm}$ on the East of Oleron island, a lower value than for $M_{2}$ by a factor 7 . The amplitude maxima of the modeled surface elevation are localized inside the Mont Saint-Michel bay, with values ranging from 5 to $75 \mathrm{~cm}$ eventually even if the amplification is localized in a smaller area than for $M_{2}$. Two amphidromic points can be seen in Fig. 9(e) near the Guernesey island $(49.5 \mathrm{~N}, 2.7 \mathrm{~W})$ and South of Brittany $(48 \mathrm{~N}, 4.7 \mathrm{~W})$. This is very different from the $M_{2}$ phase map and the propagation is cyclonic around those points. Currents associated with the propagation of the higher harmonic $M_{4}$ are plotted in Fig. 9(f). They are maximum in shallow areas as for $M_{2}$ but the orientation differs over the shelf between $45 \mathrm{~N}$ and $47.5 \mathrm{~N}$ where the currents are oriented alongshore. Therefore the $M_{4}$ harmonic does not follow its mother tide $M_{2}$ and possesses its own dynamics in shallow areas. 

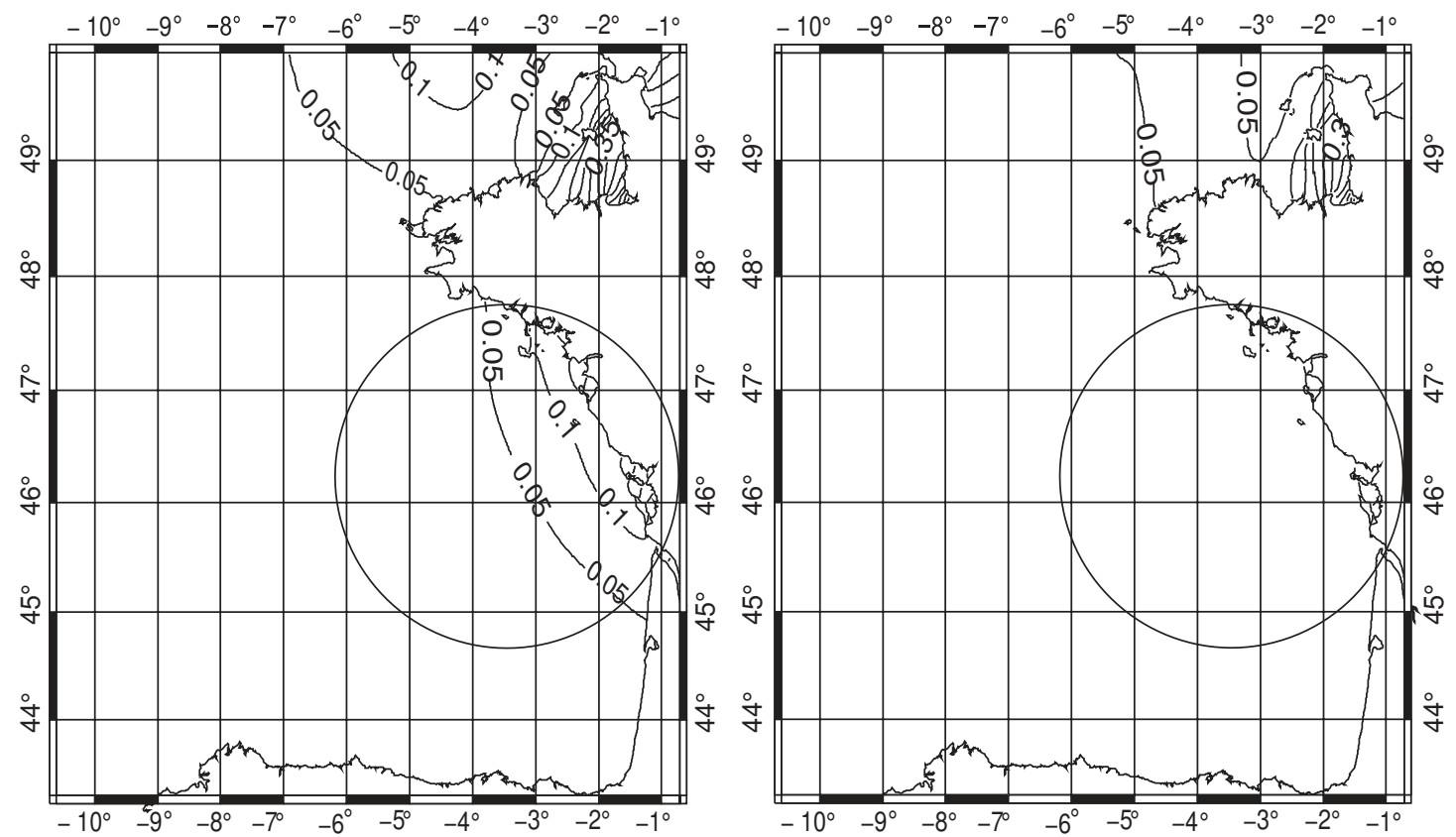

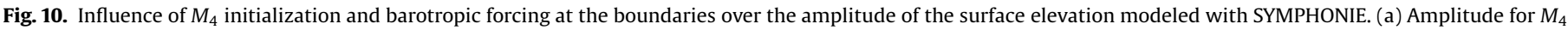

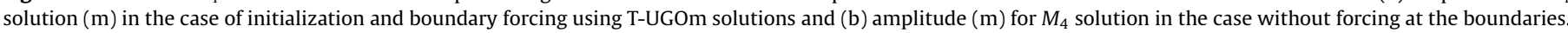
The circle indicates the region where the forcing has the stronger impact.

Table 7

RMS comparison for SYMPHONIE and T-UGOm (interpolated over a $1.5 \mathrm{~km}$ grid) tidal solutions with 53 tide gauges for $M_{2}$ and 50 for $M_{4}$ in terms of sea surface elevation

\begin{tabular}{|c|c|c|c|c|c|c|c|c|}
\hline Wave & \multicolumn{4}{|l|}{ SYMPHONIE } & \multicolumn{4}{|c|}{ T-UGOm 1.5 km } \\
\hline$M_{2}$ & 3.9 & 130.6 & 10.9 & 11.3 & 1.0 & 175.9 & 7.6 & 7.6 \\
\hline
\end{tabular}

From left to right in the table: mean complex error modulus, mean complex error phase, RMS (root mean square) for the complex error relatively to the mean, RMS for the complex error relatively to zero.

\subsection{3. $M_{4}$ amplification at the coast: sensitivity to boundary forcing}

The case of $M_{4}$ is very interesting because the first $M_{2}$ harmonic is very difficult to model and its forcing at the boundary is not straightforward as previously mentioned in Section 2.3. In particular, the English Channel located at the Eastern boundary of the modeling domain is a major generation region for $M_{4}$ (Le Provost, 1991). Following Le Cann (1990) and Alvarez et al. (1997) the energy entering the domain by the North-Eastern boundary should be responsible for the amplification at the coast in the Bay of Biscay.

A sensitivity study is thus carried out in order to study influence of the forcing over the modeled amplitudes. The difference between the cases with and without initialization and forcing by $M_{4}$ at the boundary is illustrated in Figs. 10(a) and (b). The most important differences appear at the Atlantic coast (in the circled areas). Surface elevation amplitudes in the forced case are 3 times higher than those without forcing by $M_{4}$ at the boundary in the "Pethuis Charentais", with values of 33 and $11 \mathrm{~cm}$, respectively. This means that local generation is not sufficient to explain the coastal amplification and that it is important to take the forcing into account. The question of the importance of the $M_{4}$ forcing was raised 20 years ago, and Le Cann (1990) wrote that the $M_{4}$ wave propagating outside the English Channel into the deep ocean was thought to co-oscillate with the Bay of Biscay explaining the great amplitudes observed nearshore (due to resonance), with small amplitudes at the shelf break (due to the absence of resonance).
The energy flux computed for $M_{4}$ using T-UGOm presented in Figs. 6 and 7 shows a different pattern. In the Northern part of the domain, the incoming energy flux is directed mainly Westward. If the English Channel is a strong area for $M_{4}$ generation, another important generation region is located in the Southern Atlantic off South America (i.e. the Argentina margin and the Malvine shelf). The energy flux is directed Northward after this generation in the Southern Atlantic ocean and reaches the Bay of Biscay. This energy flux is then directed cross-shore over the shelf of the Bay of Biscay toward the coastal areas of strong dissipation for $M_{4}$ as shown in Fig. 10. As a consequence there is a high sensitivity to the Southern boundary condition for the $M_{4}$ solution.

\subsection{Comparisons with observations}

In order to validate the model tidal solutions, the amplitudes and phase lags of both tidal surface elevations and currents of the forcing T-UGOm solution and the SYMPHONIE solution are compared with in situ measurements.

\subsubsection{Tidal elevations}

Comparisons have been made with tidal gauges of the Bay of Biscay. The observation network is mostly made of more than 100 years of coastal numerical recording tide gauges. The time series 
Table 8

Comparison of the tidal components $M_{2}$ and $M_{4}$ between the SYMPHONIE solution and the solution from 53 tide gauges

\begin{tabular}{|c|c|c|c|c|c|c|c|c|}
\hline \multirow[t]{2}{*}{ Station } & \multirow[t]{2}{*}{ Lat $(\mathrm{N})$} & \multirow[t]{2}{*}{ Lon (W) } & \multicolumn{3}{|l|}{$M_{2}$} & \multicolumn{3}{|l|}{$M_{4}$} \\
\hline & & & A & G & $\delta_{\text {tot }}$ & A & G & $\delta_{\text {tot }}$ \\
\hline Basse des chats & 47.58 & 3.38 & $150 / 149.9$ & $97.2 / 98.0$ & 1.4 & $7.7 / 6.5$ & $26.2 / 20.0$ & 1.0 \\
\hline Basse Royale & 48.3 & 4.83 & $194.5 / 196.8$ & 104.9/105.0 & 1.7 & $3.2 / 1.7$ & $135.3 / 234.3$ & 2.7 \\
\hline Bilbao & 43.34 & 3.04 & $132.3 / 130.3$ & $92.6 / 92.7$ & 1.4 & $2.8 / 3.0$ & $324.4 / 321.6$ & 0.2 \\
\hline Birvideaux & 47.48 & 3.28 & $124.0 / 151.0$ & $95.5 / 97.7$ & 1.5 & $8.0 / 7.1$ & $21.6 / 18.6$ & 0.7 \\
\hline Boucau & 45.51 & 1.52 & 124.0/129.2 & $95.5 / 92.2$ & 6.3 & $1.8 / 2.8$ & $314.7 / 313.1$ & 0.7 \\
\hline Bouée Castouillet & 47.32 & 2.57 & $156.9 / 159.7$ & $99.2 / 98.4$ & 2.5 & $15.6 / 14.0$ & $25.5 / 21.8$ & 1.3 \\
\hline Bouée Fromentine & 46.88 & 2.20 & $155.9 / 155.6$ & $98.0 / 95.5$ & 4.8 & $15.0 / 14.6$ & $16.9 / 8.6$ & 1.5 \\
\hline Cherbourg & 49.65 & 1.63 & $186.84 / 194.5$ & $228.8 / 237.7$ & 21.9 & $14.2 / 8.9$ & $355.2 / 54.4$ & 8.7 \\
\hline Concarneau & 47.87 & 3.92 & $147.9 / 148.7$ & $99.0 / 97.7$ & 2.6 & $6.3 / 4.7$ & $36.6 / 23.1$ & 1.4 \\
\hline Gatseau & 45.80 & 1.22 & $150.9 / 159.4$ & $98.1 / 97.4$ & 6.2 & $14.6 / 15.9$ & $343.5 / 21.7$ & 7.1 \\
\hline Gijon & 43.56 & 5.70 & 131.3/125.6 & $91.1 / 91.6$ & 4.1 & $2.1 / 2.1$ & $316.1 / 308.3$ & 0.2 \\
\hline Glénan & 47.73 & 3.95 & $149.5 / 148.2$ & $98.4 / 97.6$ & 1.7 & $6.0 / 4.5$ & $34.5 / 21.3$ & 1.4 \\
\hline Grand Trou & 47.05 & 2.63 & $159.4 / 155.6$ & $97.8 / 96.5$ & 3.7 & $12.8 / 11.8$ & $22.9 / 12.5$ & 1.7 \\
\hline Herbaudière & 47.03 & 2.3 & $167.7 / 163.6$ & $99.7 / 96.8$ & 6.6 & $18.1 / 16.3$ & $30.0 / 20.9$ & 2.3 \\
\hline Hoedic & 47.33 & 2.87 & $158.1 / 155.9$ & $98.0 / 98.8$ & 2.1 & $13.6 / 11.2$ & $25.2 / 21.9$ & 1.7 \\
\hline Houat & 47.4 & 2.95 & $155.1 / 157.7$ & 101.5/99.6 & 4.1 & $13.8 / 11.6$ & $33.0 / 26.5$ & 1.9 \\
\hline La Banche & 47.17 & 2.47 & $158.9 / 160.5$ & $100.0 / 97.9$ & 4.3 & $16.0 / 14.9$ & $27.1 / 20.3$ & 1.5 \\
\hline La Pallice & 46.17 & 1.22 & $175.9 / 68.7$ & $98.3 / 99.3$ & 5.6 & $24.8 / 23.6$ & $9.6 / 22.6$ & 4.0 \\
\hline Le Conquet & 48.37 & 4.78 & $202.5 / 203.7$ & $110.6 / 112.2$ & 4.1 & $7.0 / 6.9$ & $134.3 / 200.6$ & 5.4 \\
\hline Le Croisic & 47.30 & 2.51 & $161.8 / 160.0$ & $102.1 / 98.3$ & 7.5 & $16.8 / 14.3$ & $33.3 / 21.8$ & 2.8 \\
\hline Le Palais & 47.35 & 3.15 & $155.1 / 156.0$ & $98.6 / 98.4$ & 0.7 & $10.9 / 9.7$ & $24.9 / 20.9$ & 1.0 \\
\hline Sables d'olonnes & 46.5 & 1.82 & $156.3 / 151.2$ & $97.3 / 95.8$ & 4.6 & $15.2 / 12.8$ & 4.9/359.6 & 2.0 \\
\hline Loctudy & 47.83 & 4.17 & $147.6 / 148.4$ & $95.0 / 97.7$ & 4.9 & $4.9 / 3.8$ & $23.0 / 23.5$ & 0.8 \\
\hline Pornichet & 47.25 & 2.33 & $163.4 / 163.2$ & $99.0 / 99.4$ & 0.8 & $18.0 / 16.9$ & $29.4 / 26.3$ & 1.0 \\
\hline Port-Louis & 47.7 & 3.35 & $146.5 / 150.6$ & $100.8 / 98.6$ & 5.0 & $7.6 / 7.0$ & $26.0 / 23.2$ & 0.5 \\
\hline Port Jointville & 46.73 & 2.35 & $153.4 / 152.2$ & $98.7 / 94.9$ & 7.4 & $12.9 / 11.4$ & $12.2 / 1.5$ & 1.9 \\
\hline Port-Tudy & 47.65 & 3.45 & $149.8 / 150.0$ & $98.9 / 98.0$ & 1.5 & $7.3 / 6.2$ & $29.4 / 20.2$ & 1.1 \\
\hline Royan & 45.6 & 1.02 & $148.2 / 152.2$ & 108.8/105.6 & 6.5 & $7.6 / 2.7$ & $359.7 / 26.5$ & 3.8 \\
\hline Saint-Hélier Jersey & 49.18 & 2.11 & $333.2 / 349.2$ & $182.2 / 198.8$ & 70.6 & $19.1 / 24.7$ & $300.5 / 356.1$ & 14.8 \\
\hline Saint-Gildas & 47.13 & 2.25 & $168.4 / 165.5$ & $100.6 / 98.9$ & 4.0 & $19.0 / 18.5$ & $30.8 / 26.5$ & 1.0 \\
\hline Saint-Guenole & 47.82 & 4.37 & $150.6 / 151.0$ & $96.5 / 95.7$ & 1.4 & $3.9 / 1.9$ & 47.0/29.9 & 1.5 \\
\hline Saint-Nazaire & 47.27 & 2.2 & 175.0/169.1 & $104.1 / 100.7$ & 8.2 & $20.0 / 20.5$ & $44.7 / 35.0$ & 2.4 \\
\hline Santander & 43.46 & 3.79 & $133.7 / 128.8$ & $97.5 / 92.3$ & 9.0 & $2.4 / 2.5$ & $335.5 / 316.0$ & 0.6 \\
\hline Vilaine-P1 & 47.43 & 3.18 & $150.3 / 153.2$ & $100.3 / 97.9$ & 4.8 & $8.7 / 8.2$ & $28.8 / 19.2$ & 1.1 \\
\hline Vilaine-P2 & 47.23 & 2.93 & $151.4 / 153.6$ & $100.3 / 97.9$ & 4.7 & $10.4 / 10.0$ & $26.1 / 17.7$ & 1.1 \\
\hline COURIR1 & 47.85 & 5.97 & $147.7 / 149.4$ & $105.8 / 105.7$ & 1.3 & $1.4 / 1.8$ & $196.4 / 257.3$ & 1.2 \\
\hline COURIR3 84-3 & 48.02 & 6.22 & $150.9 / 150.2$ & $107.1 / 108.5$ & 2.5 & $0.6 / 2.1$ & $196.3 / 251.4$ & 1.3 \\
\hline COURIR5 2 & 47.83 & 5.78 & $141.0 / 139.0$ & $99.4 / 100.8$ & 2.8 & $0.6 / 1.5$ & $283.5 / 276.8$ & 0.7 \\
\hline COURIR5 3 & 47.27 & 6.0 & 130.7/129.6 & $97.7 / 98.9$ & 2.0 & $1.0 / 1.4$ & $320.8 / 309.9$ & 0.3 \\
\hline G & 49.62 & 8.62 & $137.8 / 133.4$ & 121.6/124.6 & 5.9 & $2.7 / 2.7$ & $231.0 / 235.3$ & 0.2 \\
\hline Gaillouneys & 44.57 & 1.23 & $125.8 / 131.2$ & $102.2 / 92.1$ & 16.5 & $1.5 / 3.7$ & 29.0/313.8 & 2.6 \\
\hline Gastom-M1P1 & 47.52 & 6.8 & $126.8 / 127.5$ & $101.4 / 100.8$ & 1.0 & $1.4 / 1.4$ & $310.3 / 301.9$ & 0.1 \\
\hline $\mathrm{H}$ & 48.92 & 9.35 & $123.2 / 120.2$ & $112.4 / 113.0$ & 2.3 & $1.5 / 1.7$ & $267.1 / 275.4$ & 0.2 \\
\hline I & 47.85 & 10.38 & 108.7/109.1 & 106.7/103.9 & 3.8 & $0.8 / 1.0$ & $304.8 / 301.1$ & 0.1 \\
\hline IAPSO.1.1.7 & 47.45 & 8.43 & 122.0/118.6 & $102.0 / 101.5$ & 2.4 & $0.7 / 1.1$ & $210.0 / 303.5$ & 1.0 \\
\hline IAPSO.1.1.11 & 48.62 & 9.7 & $114.8 / 114.5$ & $107.0 / 108.0$ & 1.4 & & & \\
\hline IAPSO.1.1.85 & 48.13 & 8.08 & $124.3 / 124.1$ & $106.0 / 105.8$ & 0.4 & & & \\
\hline IAPSO.1.1.59 & 48.8 & 7.02 & $149.9 / 150.2$ & 117.0/119.5 & 4.7 & & & \\
\hline $\mathrm{M} 1^{\prime}$ & 45.08 & 2.43 & 136.0/131.2 & $93.2 / 93.3$ & 3.4 & $2.8 / 2.8$ & $338.2 / 319.8$ & 0.6 \\
\hline $\mathrm{M} 2^{\prime}$ & 45.52 & 3.42 & 134.2/129.9 & $94.8 / 94.2$ & 3.2 & $2.6 / 2.3$ & $339.8 / 320.8$ & 0.6 \\
\hline M3' & 46.51 & 4.52 & $135.8 / 131.3$ & $97.4 / 96.0$ & 3.9 & $1.6 / 1.9$ & $348.3 / 327.7$ & 0.5 \\
\hline Manmod-P2 & 49.12 & 5.68 & $185.4 / 180.3$ & $127.1 / 131.6$ & 10.6 & $6.4 / 5.6$ & 149.2/190.2 & 3.0 \\
\hline MGFCOR large & 46.32 & 5.4 & $130.2 / 127.6$ & $96.8 / 96.2$ & 2.0 & $1.8 / 1.7$ & $334.7 / 318.0$ & 0.4 \\
\hline
\end{tabular}

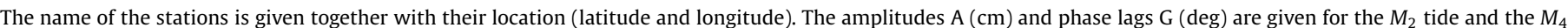

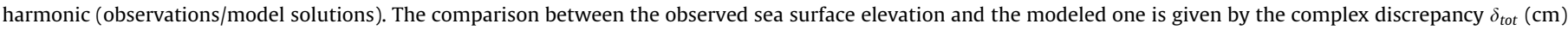
that is the complex error norm times $\frac{1}{\sqrt{2}}$. The horizontal line separates the coastal locations and the deep ocean locations.

analysis is summarized for $M_{2}$ and $M_{4}$ in Table 7 . This table is dedicated to a statistical comparison between solutions from SYMPHONIE, T-UGOm and tide gauges. Details on the SYMPHONIE and tide gauges solutions at each location are available in Table 8 and in Fig. 11.

Let $\eta_{\text {sym }}^{i}$ be the surface elevation of a partial tide modeled by SYMPHONIE, with $i$ the subscript for the comparison position $\left(\eta_{o b s}^{i}\right.$ stands for the corresponding observation).

The complex mean error is $\bar{e}=(1 / n) \sum_{i=1}^{n} \eta_{o b s}^{i}-\eta_{\text {sym }}^{i}=$ $(1 / n) \sum_{i=1}^{n} e_{i}$. It is split into a real part and a complex part with amplitude $A_{e r r}$ and phase $G_{e r r}$. The complex mean error modulus $\operatorname{Mod}_{\text {err }}$ in the table corresponds to $(1 / \sqrt{2}) A_{\text {err }}$ and the RMS (root mean square) error is also given. The RMS of the complex error relatively to the mean value is

$\sqrt{\frac{\sum_{i}\left|e_{i}-\bar{e}\right|^{2}}{2 n}}$

From Table 7 it appears that the solutions of the two models differ from the observations in the same way. For the semi-diurnal 


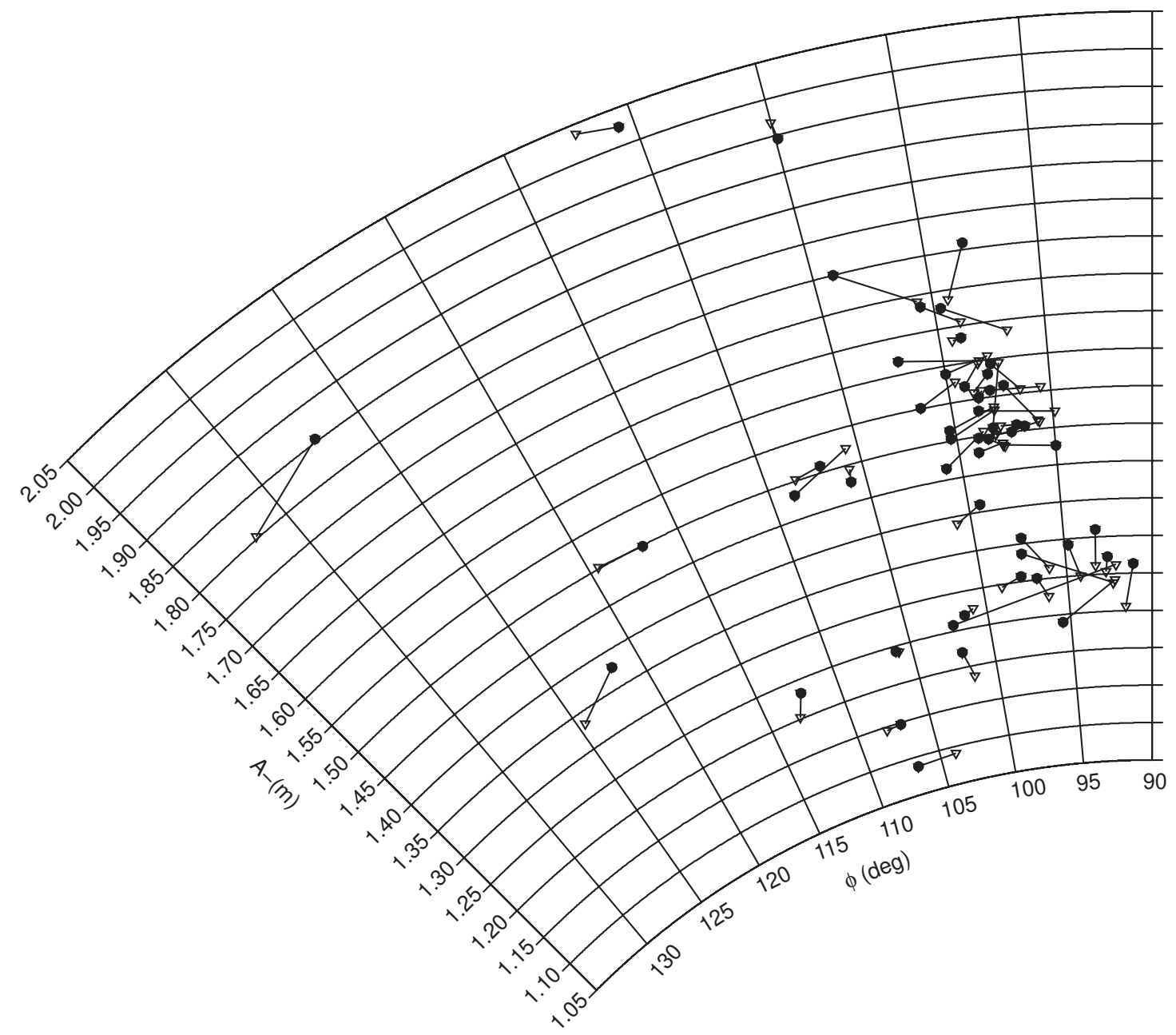

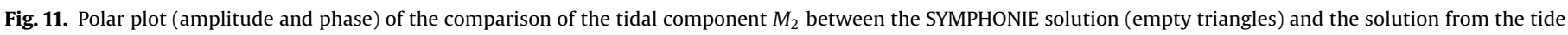
gauges (full circles).

tide $M_{2}$, that is the major constituent of the global tidal signal, the complex RMS error is $11.3 \mathrm{~cm}$ for the SYMPHONIE solution against $7.6 \mathrm{~cm}$ for the T-UGOm solution interpolated on the SYMPHONIE run grid (that is about $30 \%$ less). The SYMPHONIE solution is therefore not as good as the T-UGOm solution in terms of surface elevation. The mean complex error modulus is $3.9 \mathrm{~cm}$ for the SYMPHONIE solution, much more than the $1.0 \mathrm{~cm}$ obtained for the T-UGOm solution. This implies a RMS error relative to the origin bigger than the RMS error spreading relative to the mean $(10.9 \mathrm{~cm}$ for the SYMPHONIE solution). However, Fig. 11 shows that errors are due to phase differences more than differences in amplitudes between the model and the tide gauges solutions.

For the $M_{4}$ harmonic, the solutions from both models have globally the same behavior. The error is nearly the same for the SYMPHONIE solution and the T-UGOm solution, with an equal modulus of $0.7 \mathrm{~cm}$. But errors are more spread over with the SYMPHONIE simulation.

The explanations for the differences between the model and the forcing solutions depend upon the region of interest. They are largely due to differences in grids (namely the finite-difference structured grids, with a horizontal resolution of $1.5 \mathrm{~km}$, versus the finite-element unstructured grids that are locally refined, with a horizontal resolution spaning from $20 \mathrm{~km}$ in the deep ocean to less than $1 \mathrm{~km}$ at the coast) with the associated bathymetry resolution and also to the analysis duration and forcing of the model. For the T-UGOm solution 40 tidal constituents are analyzed over one year against five components during two months for the SYMPHONIE solution.

The errors associated with the sea surface elevation modeled by SYMPHONIE are spread over a large range of values. They reach their maximum for the tide gauges located near the coasts (see Table 8). The difference in amplitude is $0.7 \mathrm{~cm}$ at station GASTOMM1P1 located above the shelf break, but it reaches $16 \mathrm{~cm}$ at SaintHélier for $M_{2}$. In order to take the phase lag into account, a look at the associated complex discrepancy shows a greater difference with values ranging from $1 \mathrm{~cm}$ at GASTOM-M1P1 to $70 \mathrm{~cm}$ at Saint-Hélier. Locally, at station Manmod-P2, the complex discrepancy is more than $10 \mathrm{~cm}$ even over the shelf break. This is probably due to a poor resolution and bathymetry representation in the model in this region of rapidly varying depth.

The poor quality of the modeled solution at Saint-Hélier can have several origins. First, the station is located in a harbor, with rapidly varying depth. Then, the version of the SYMPHONIE model used here was barotropic. It does not take into account the 3D effects on the modeled tide whereas the T-UGOm model includes the dissipation process of barotropic to baroclinic energy transfer due to internal wave generation over topographic features (Carrere and Lyard, 2003). Moreover, a comparison with the TUGOm solution at Saint-Hélier has shown that the grid mesh of the model does not allow it to be as accurate as T-UGOm in various areas (mostly near the coasts) because of the use of a regular grid instead of a finite-element one. 

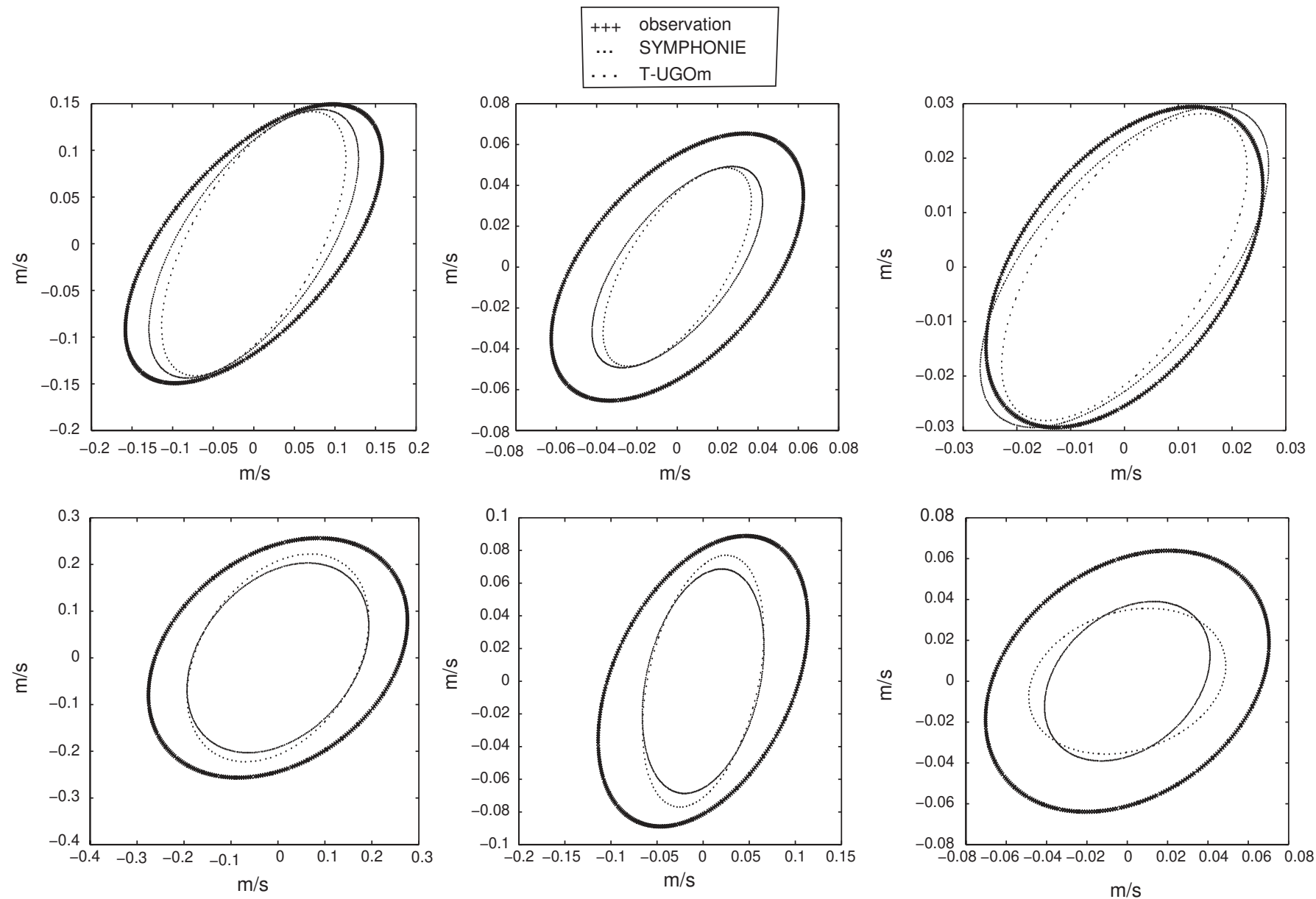

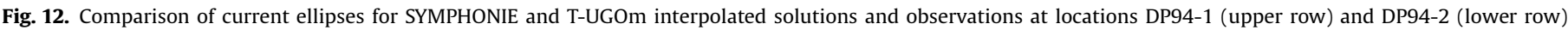
(Perenne and Pichon, 1999; Pichon and Correard, 2006) for $M_{2}, S_{2}$ and $N_{2}$ waves (in this order from left to right.

Table 9

Comparison of current ellipses parameters for models and observations at locations DP94-1 and DP94-2 from Pichon and Correard (2006)

\begin{tabular}{|c|c|c|c|c|c|c|}
\hline Location & Data & Tidal constituent & Semi-major axis $\left(\mathrm{cm} \mathrm{s}^{-1}\right)$ & Semi-minor axis $\left(\mathrm{cm} \mathrm{s}^{-1}\right)$ & Orientation (deg) & Situation (deg) \\
\hline \multirow[t]{9}{*}{ DP94-1 } & \multirow[t]{3}{*}{ Observations } & $M_{2}$ & 19.6 & -9.5 & 42.3 & 76.9 \\
\hline & & $S_{2}$ & 7.9 & -4.3 & 47.4 & 124.2 \\
\hline & & $\mathrm{N}_{2}$ & 3.4 & -1.9 & 52.5 & 71.7 \\
\hline & \multirow[t]{3}{*}{ SYMPHONIE } & $M_{2}$ & 17.5 & -8.2 & 49.8 & 74.7 \\
\hline & & $S_{2}$ & 5.9 & -2.7 & 51.7 & 115.0 \\
\hline & & $\mathrm{N}_{2}$ & 3.6 & -1.7 & 49.2 & 53.3 \\
\hline & \multirow[t]{3}{*}{ T-UGOm } & $M_{2}$ & 16.5 & -7.5 & 54.6 & 72.5 \\
\hline & & $S_{2}$ & 5.6 & -2.4 & 56.6 & 113.3 \\
\hline & & $N_{2}$ & 3.3 & -1.5 & 54.2 & 51.6 \\
\hline \multirow[t]{9}{*}{ DP94-2 } & \multirow[t]{3}{*}{ Observations } & $M_{2}$ & 30.6 & -22.0 & 38.4 & 89.9 \\
\hline & & $S_{2}$ & 12.3 & -7.5 & 29.1 & 139.4 \\
\hline & & $\mathrm{N}_{2}$ & 7.7 & -5.6 & 36.0 & 64.4 \\
\hline & \multirow[t]{3}{*}{ SYMPHONIE } & $M_{2}$ & 22.9 & -16.2 & 49.2 & 42.6 \\
\hline & & $S_{2}$ & 7.7 & -5.6 & 48.7 & 84.5 \\
\hline & & $\mathrm{N}_{2}$ & 4.6 & -3.3 & 40.5 & 27.0 \\
\hline & \multirow[t]{3}{*}{ T-UGOm } & $M_{2}$ & 24.5 & -16.4 & 55.7 & 38.2 \\
\hline & & $S_{2}$ & 8.5 & -5.5 & 56.3 & 78.6 \\
\hline & & $\mathrm{N}_{2}$ & 5.0 & -3.4 & 53.5 & 17.0 \\
\hline
\end{tabular}

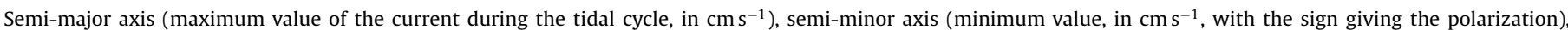

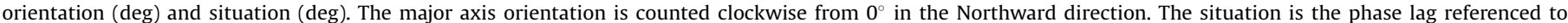
$\mathrm{UT}+0$.

The SYMPHONIE model is thus able to correctly represent the tides in the Bay of Biscay and to follow the initial and forcing solutions, with a tendency to over-amplification of the tides near the coast. This solution is a little poorer than that found with T-UGOm, with problems of resolutions and local topography errors in models enhanced in SYMPHONIE. But the comparison 
above the shelf break is very satisfying, hence this model is well suited to the present problem, because this is where internal tide generation takes place.

\subsubsection{Tidal currents}

Because mean currents (on a vertical plane) are obtained with a barotropic model, the comparison with observations is not obvious. Actually computing the mean currents from in situ measurements that include baroclinicity is very difficult, and the comparison presented hereafter has to be regarded as essentially qualitative.

The current ellipse parameters for the modeled solutions and for the MINT94 observed solutions are compared for the semi-diurnal components $M_{2}, S_{2}$ and $N_{2}$ of the tidal spectrum. Two moorings are considered, at stations DP94-1 $(48.109 \mathrm{~N}$, 8.485W) and DP94-2 (47.239N, 5.942W) (see Fig. 8), with current meters (ADCP) at $300 \mathrm{~m}$ depth (and 30 layers on the vertical). They were active in 1994 during one month when the seasonal thermocline was formed, under conditions of weak stratification, from mid-April to mid-May for DP94-1 and from mid-May to midJune for DP94-2 (Perenne and Pichon, 1999). The observed barotropic current is defined as the vertical average of the measured current at each level and the tidal constituents are extracted with harmonic analysis. At station DP94-2, the slope is very steep (about 10\%) and the horizontal variability of the currents is maximum (Huet, 1999). Therefore, a quantitative comparison with model solutions at that location seems particularly difficult.

The comparison has been carried out by choosing a depth criterion of $300 \mathrm{~m}$ for the model locations. Results are summarized in Fig. 12 and in Table 9.

The first comparison is made between the SYMPHONIE solution and the T-UGOm interpolated solution. As for the surface elevation, the two solutions are very close to each other, at both DP94-1 and DP94-2 locations (Fig. 12). The SYMPHONIE model tends to overestimate the amplitude of the current for the three waves $M_{2}, S_{2}$ and $N_{2}$ at DP94-1 compared with the forcing solution, with a mean of $0.5 \mathrm{~cm} \mathrm{~s}^{-1}$ for the semi-major axis and $0.4 \mathrm{~cm} \mathrm{~s}^{-1}$ for the semi-minor axis; by contrast, at DP94-2 it tends to underestimate this amplitude. As the SYMPHONIE solutions globally follow the forcing, errors from the observations may originate from errors in the forcing.

At DP94-1, the SYMPHONIE modeling correctly describes the observed currents for $M_{2}, S_{2}$ and $N_{2}$, with an underestimation of $2 \mathrm{~cm} \mathrm{~s}^{-1}$ for the $M_{2}$ and $S_{2}$ semi-major axes and an overestimation of $0.2 \mathrm{~cm} \mathrm{~s}^{-1}$ for the $N_{2}$ axis. The same tendency appears for the semi-minor axis. Locally, the bathymetry gradient is oriented North-South and the current ellipse makes an angle of nearly $45^{\circ}$ with this direction, the difference going from 1 to $8^{\circ}$. The phase lag bias is only 5 min for $M_{2}$ at DP94-1. Thus the comparison at DP94-1 location is satisfying, with the SYMPHONIE solution fitting better the observation than the T-UGOm interpolated solution.

At DP94-2, on the contrary, the T-UGOm interpolated solution fits better the data from the mooring. Both the solutions from SYMPHONIE and T-UGOm show larger errors than at DP94-1. The bias in amplitude is $7.68 \mathrm{~cm} \mathrm{~s}^{-1}$ for the SYMPHONIE solution ( $6 \mathrm{~cm} \mathrm{~s}^{-1}$ for the T-UGOm one) for the $M_{2}$ semi-major axis. The orientations of the modeled ellipses are close to the observed ones, but the observed phase lag is twice the modeled one.

It is important to keep in mind the resolution and bathymetry limitations of the models, so that poor bathymetry representation could explain the differences at DP94-2 station. Another point to consider is the analysis. We took only five waves in order to separate the components well, but the observations are sampled over one month only, meaning that the treatment must be an admittance method in order to get all the waves $\left(M_{2}, S_{2}, N_{2}\right.$ and $K_{2}$ ). The differences in post-treatment may also induce bias. But from this localized comparison, the SYMPHONIE modeling is competitive with a regional T-UGOm modeling dedicated to tidal studies (with bias from observations of the same order of magnitude). This is of primary importance as the tidal current is a forcing for internal tide generation at the shelf break in the Bay of Biscay.

\section{Discussion}

Significant progress has been made during the last 15 years in our knowledge of deep ocean and coastal tides with the emergence of altimetry. But as highlighted by Lyard et al. (2006) the spectral approach has reached its limits with the FES2004 atlas. The regional hydrodynamic model T-UGOm is a new generation model that solves the nonlinear dynamics and the related NEA-2004 tidal atlas shows a great improvement of the tidal solution in coastal areas. This yields an improvement of the embedded SYMPHONIE solution at a regional/coastal scale.

The comparisons between the NEA-2004 atlas, the SYMPHONIE solution and measurements show a good agreement between the model solutions and observations. They demonstrate the ability for the models to accurately represent the barotropic tides in the Bay of Biscay, with bathymetry and boundary conditions as the major sources of error. This study also illustrates the need to take the $M_{4}$ harmonic forcing into account at the regional model boundary, with errors of more than $100 \%$ observed near the coasts of the Bay of Biscay depending on this forcing. For a realistic representation of the harmonic, forcing at both the Northern and the Southern boundaries of the Bay of Biscay model is a prerequisite, as a great part of energy at the $M_{4}$ frequency propagates from the Southern Atlantic ocean before entering the bay.

Barotropic tides in the Bay of Biscay are very strong and a large amount of energy is dissipated over the continental shelf, especially at the English Channel entrance. The most energetic component is the $M_{2}$ wave which represents more than $80 \%$ of the total energy in the European shelf seas. As this work deals with the coastal zone, a special attention is paid to nonlinearities affecting the tidal spectrum. The energy budget is addressed in terms of a spectral energy decomposition, including spectral mean energy fluxes plus cross terms. This yields to $250 \mathrm{GW}$ for $\mathrm{M}_{2}$ and $1 \mathrm{GW}$ for $M_{4}$. This last value may be underestimated due to a damped tidal amplitude for the $M_{4}$ NEA-2004 solution.

Finally, this validation of the barotropic tides is of primary importance for the modeling of internal tides. Indeed, internal tides are generated at the vicinity of the shelf break and arise from the interaction of tidal currents with bathymetry as will be described in the next part of the paper (Pairaud et al., 2008), dedicated to internal tides in the Bay of Biscay.

\section{Acknowledgments}

This study was funded by a PATOM 2005 project. The authors want to thank David Greenberg for providing us with the tools used to make polar plots and the computing team of the Aerologie Laboratory of Toulouse for their help with 64 bit computers. They are also grateful to the members of the Coastal Oceanography group of Toulouse for the constructive discussions (http:// poc.obs-mip.fr) and to Bruno Voisin for his help in improving the English of the paper. Thanks are extended to Alan Davies and anonymous reviewers for their comments and suggestions on the original manuscript. 


\section{Appendix A. Tidal forcing in the SYMPHONIE modeling}

Tidal forcing consists first of tidal surface elevations and currents introduced as forcing terms in the boundary conditions (35), and secondly of astronomical and loading potentials providing, through the horizontal components of their gradients, a barotropic force added to the momentum equation. Tidal sea surface elevations and currents have the following forms:

$$
\begin{aligned}
\eta(\lambda, \varphi, t)= & \sum_{k} f_{k} \eta_{0, k}(\lambda, \varphi) \cos \left(\omega_{k}\left(t-t_{0}\right)\right. \\
& \left.+V_{0, k}+u_{k}-G_{\eta, k}(\lambda, \varphi)\right) \\
u(\lambda, \varphi, t)= & \sum_{k} f_{k} u_{0, k}(\lambda, \varphi) \cos \left(\omega_{k}\left(t-t_{0}\right)\right. \\
& \left.+V_{0, k}+u_{k}-G_{u, k}(\lambda, \varphi)\right)
\end{aligned}
$$

$$
\begin{aligned}
v(\lambda, \varphi, t)= & \sum_{k} f_{k} v_{0, k}(\lambda, \varphi) \cos \left(\omega_{k}\left(t-t_{0}\right)\right. \\
& \left.+V_{0, k}+u_{k}-G_{v, k}(\lambda, \varphi)\right)
\end{aligned}
$$

where the subscript $k$ stands for the partial tides taken into account. $\left(\eta_{0}, u_{0}, v_{0}\right)$ and $\left(G_{\eta}, G_{u}, G_{v}\right)$ are, respectively, the amplitudes and phase lags for sea surface elevations and currents given by the NEA-2004 atlas (this issue) and depending on the longitude $\lambda$ and the latitude $\varphi$. The nodal factors $f_{k}$ and $u_{k}$ (Doodson, 1927) are taken as constant (although they are in fact slowly varying with time), $V_{0}$ is a constant related to the reference time $t_{0}$ and $\omega$ is the considered tidal frequency. Practically, we have added five of the most significant tidal waves in the Bay of Biscay $\left(M_{2}, S_{2}, N_{2}\right.$, $K_{1}$ and $M_{4}$ ). The tidal potential due to astronomical effects (Hendershott, 1972; Apel, 1987) is given by

$$
\begin{aligned}
\Pi_{A}= & \left(1+k_{2}+h_{2}\right) f a \\
& \left.\times v_{0} \frac{1-3 \sin ^{2}(\varphi)}{2}+v_{1} \sin (2 \varphi)+v_{2} \cos ^{2}(\varphi)\right) \\
& \times \cos \left(\omega\left(t-t_{0}\right)+v \lambda+V_{0}+u\right)
\end{aligned}
$$

where $a$ stands for the equilibrium amplitude of the tide and $k_{2}$ and $h_{2}$ are the Love numbers. The values for $v$ and $\left(v_{0}, v_{1}, v_{2}\right)$ depend on the nature of the tide. For the long period tide, $v=0$ and $v_{0}=1$, for the diurnal tide $v=1$ and $v_{1}=1$ and for the semidiurnal tide $v=2$ and $v_{2}=1$.

We also take into account a LSA potential $\Pi_{L}$, which can be seen as a retroaction effect of tides on themselves and are provided by the FES99 solution (Lefevre et al., 2002):

$\Pi_{L}=f \Pi_{0}(\lambda, \varphi) \cos \left(\omega\left(t-t_{0}\right)+G_{\Pi}(\lambda, \varphi)+V_{0}+u\right)$

Frequencies and associated constants in Eqs. (4)-(6) are summarized in Table 6 following computations from Schureman (1958).

Finally the hydrostatic pressure force appearing in the horizontal momentum equation is given by

$-\frac{\nabla p}{\rho_{0}}=-\frac{g}{\rho_{0}} \nabla \int_{z}^{\eta}\left(\rho-\rho_{0}\right) \mathrm{d} z^{\prime}-g \nabla\left(\eta-\Pi_{A}-\Pi_{L}\right)$

where $\rho$ and $\rho_{0}$ are, respectively, the density and a constant density associated to the Boussinesq approximation.

\section{References}

Alvarez, E., Pérez, B., Rodriguez, I., 1997. A description of the tides in the Eastern North Atlantic. Progress in Oceanography 40, 217-244.

Andersen, O.B., 1999. Shallow water tides in the northwest European shelf region from Topex/Poseidon altimetry, Journal of Geophysical Research 104 (C4), 7729-7741.

Apel, J.R., 1987. Principles of Ocean Physics. International Geophysics Series, vol. 38. Academic Press, London, 634pp.

Arakawa, A., Suarez, M.J., 1983. Vertical differencing of the primitive equation in sigma coordinates. Monthly Weather Review 111, 34-45.

Baines, P.G., 1982. On internal tide generation models. Deep-Sea Research 29, 307-338.
Battisti, D.S., Clarke, A.J., 1982a. A simple method for estimating barotropic tidal currents on continental margins with specific application to the M2 tide off the Atlantic and Pacific coasts of the United States. Journal of Physical Oceanography $12,8-16$.

Battisti, D.S., Clarke, A.J., 1982b. Estimation of nearshore tidal currents on non-smooth continental shelves. Journal of Geophysical Research 87 (C10), 7873-7878.

Bell, T.H., 1975. Topographically generated internal waves in the open ocean. Journal of Geophysical Research 80 (3), 320-338.

Carrere, L., Lyard, F., 2003. Modeling the barotropic response of the global ocean to atmospheric wind and pressure forcing. Geophysical Research Letters 30, 1275.

Cartwright, D.E., 1977. Oceanic tides. Report on Progress in Physics 40, 665-708.

Clarke, A.J., 1991. The dynamics of barotropic tides over the continental shelf and slope. In: Parker, B.B. (Ed.), Tidal Hydrodynamics. Wiley, New York, pp. 79-108.

Clarke, A.J., Battisti, D.S., 1981. The effect of continental shelves on tides. Deep-Sea Research 28, 6665-6682.

Davies, A.M., Kwong, S.C.M., 2000. Tidal energy fluxes and dissipation on the European continental shelf. Journal of Geophysical Research 105 (C9), 21969-21989.

Doodson, A.T., 1927. The analysis of tidal observations. Philosophical Transactions of the Royal Society of London 227, 223-279.

Egbert, G.D., Ray, R.D., Bills, B.G., 2004. Numerical modeling of the global semidiurnal tide in the present day and in the last glacial maximum. Journal of Geophysical Research 109 (C3).

Fang, Z., Ye, A., Fang, G., 1991. Solutions of tidal motions in a semi-closed rectangular gulf with open boundary condition specified. In: Parker, B.B. (Ed.), Tidal Hydrodynamics. Wiley, New York, pp. 153-168.

Flather, R.A., 1976. A tidal model of the northwest European continental shelf. Mémoires de la Société Royale des Sciences de Liège 10,141-164.

Garrett, C., Kunze, E., 2007. Internal tide generation in the deep ocean. Annual Review of Fluid Mechanics 39 (1), 57-87.

Hendershott, M.C., 1972. The effects of solid earth deformations on global ocean tides. Geophysical Journal of the Royal Astronomical Society 29, 399-402.

Huet, P., 1999. Validation et mise en valeur des résultats de marée interne. EPSHOM Brest, Report No. 25.

Jezequel, N., Maze, R., 2001. A barotropic model of the linear semidiurnal tide over a continental slope. Deep-Sea Research 48, 1375-1399.

Le Cann, B., 1990. Barotropic tidal dynamics of the Bay of Biscay shelf: observations, numerical modelling and physical interpretation. Continental Shelf Research 10, 723-758.

Lefevre, F., Lyard, F., Le Provost, C., Schrama, E.J.O., 2002. Fes99: a global tide finite element solution assimilating tide gauge and altimetric observation. Journal of Atmospheric and Oceanic Technology 19, 1345-1356.

Le Provost, C., 1991. Generation of overtides and compound tides (review). In: Parker, B.B. (Ed.), Tidal Hydrodynamics. Wiley, New York, pp. 269-295, 883pp.

Le Provost, C., Fornerino, M., 1985. Tidal spectroscopy of the English Channel with a numerical model. Journal of Physical Oceanography 15, 1009-1031.

Le Provost, C., Lyard, F., 1998. Energetics of the semi-diurnal M2 ocean tide. Progress in Oceanography 40, 37-52.

Le Provost, C., Rougier, G., Poncet, A., 1981. Numerical modeling of the harmonic constituents of the tides, with application to the English Channel. Journal of Physical Oceanography 11 (8), 1123-1138.

Le Provost, C., Genco, M.L., Lyard, F., Vincent, P., Canceil, P., 1994. Tidal spectroscopy of the world ocean tides from a finite element hydrodynamic model. Journal of Geophysical Research 99 (C12), 24777-24798.

Lyard, F., Lefevre, F., Letellier, T., Francis, O., 2006. Modelling the global ocean tides: modern insights from Fes2004. Ocean Dynamics 56, 394-415.

Lynch, D.R., Gray, W.G., 1979. A wave equation model for finite element model for finite element tidal computations. Computers and Fluids 7, 207-228.

Marsaleix, P., Auclair, F., Estournel, C., 2006. Considerations on open boundary conditions for regional and coastal ocean models. Journal of Atmospheric and Oceanic Technology 23 (11), 1604-1613.

Marsaleix, P., Auclair, F., Floor, J.W., Herrmann, M.J., Estournel, C., Pairaud, I., Ulses, C., 2008. Energy conservation issues in sigma-coordinate free-surface ocean models. Ocean Modelling 20, 61-89.

Munk, W., Wunsch, C., 1998. Abyssal recipes II: energetics of tidal and wind mixing. Deep-Sea Research 45, 1976-2000.

Munk, W.H., Cartwright, D.E., 1966. Tidal spectroscopy and prediction. Philosophical Transactions of the Royal Society of London A 259, 533-581.

Pairaud, I., Auclair, F., Lyard, F., Marsaleix, P., Pichon, A., 2008. Dynamics of the semi-diurnal and quarter-diurnal tides in the Bay of Biscay. Part 2: baroclinic tides. Continental Shelf Research, submitted for publication.

Perenne, N., Pichon, A., 1999. Effect of barotropic tidal rectification on lowfrequency circulation near the shelf break in the northern Bay of Biscay. Journal of Geophysical Research 104, 13489-13506.

Pichon, A., Correard, S., 2006. Internal tides modelling in the Bay of Biscay. Comparisons with observations. Scientia Marina 70S1, 68-88.

Pingree, R.D., Mardell, G.T., New, A.L., 1986. Propagation of internal tides from the upper slopes of the Bay of Biscay. Nature 321,154-158.

Pugh, D.T., 1989. Tide, Surges and Mean Sea-Level. Wiley, New York.

Schureman, P., 1958. Manual of Harmonic Analysis and Prediction of Tides. Special Publication 98. U.S. Government Printing Office.

Simmons, H.L., Jayne, S.R., St Laurent, L.C., Weaver, A.J., 2004. Tidally driven mixing in a numerical model of the ocean general circulation. Ocean Modelling 405, 775-778.

Smagorinsky, J., 1993. Some historical remarks on the use of nonlinear viscosities. In: Galperin, B., Orszag, S. (Eds.), Large Eddy Simulations of Complex Engineering and Geophysical Flows. Cambridge University Press, Cambridge, UK, pp. 4-36. 